\title{
CLASSIFICATION IN CHAINS OF THREE-DIMENSIONAL REAL EVOLUTION ALGEBRAS
}

\author{
B. A. NARKUZIYEV, U.A. ROZIKOV
}

\begin{abstract}
A chain of evolution algebras (CEA) is an uncountable family (depending on time) of evolution algebras on the field of real numbers. The matrix of structural constants of a CEA satisfies Kolmogorov-Chapman equation. In this paper, we consider three CEAs of three-dimensional real evolution algebras. These CEAs depend on several (non-zero) functions defined on the set of time. For each chain we give full classification (up to isomorphism) of the algebras depending on the time-parameter. We find concrete functions ensuring that the corresponding CEA contains all possible three-dimensional evolution algebras.
\end{abstract}

\section{INTRODUCTION}

An evolution algebra (EA) (see [22]) is an abstract system, it gives an insight for the study of non-Mendelian genetics. In the relation between EAs and Markov processes, the Chapman-Kolmogorov equation gives the fundamental relationship between the probability transitions (kernels). There are many recent papers devoted to the theory of evolution algebras (see for example [1], [2], [12, [13, [17, [19] and the references therein).

In [3] a notion of a chain of evolution algebras (CEA) is introduced. Later, in [8] and [9] (see also [19]) the notion of CEA was generalized and a concept of flow of arbitrary finitedimensional algebras is introduced. It is known that each EA is determined by a quadratic matrix of structural constants. A CEA is a (uncountable) family of EAs depending on the two-dimensional time $(s, t), 0 \leq s \leq t$.

The matrices of structural constants (depending on $(s, t)$ ) of a CEA satisfy the ChapmanKolmogorov equation. In other words, a CEA is a continuous-time dynamical system which in a fixed time is an EA.

In [18] a wide class of finite-dimensional CEAs is constructed. In [3]-[6], [8]-[11, [14]-[16], [18]-21] several new classes of CEAs and flows of algebras are given. These investigations are used to develop the theory of Markov processes of cubic matrices [4], [11. The recent book [19] contains a systematic presentation of these algebraic and probabilistic approaches to study population dynamics.

In this paper we consider three CEAs constructed in [6].

Before formulation of our main problem, let us give basic notations. Following [3] we consider a family $\left\{E^{[s, t]}: s, t \in \mathbb{R}, 0 \leq s \leq t\right\}$ of $n$-dimensional evolution algebras over the

2010 Mathematics Subject Classification. 17D92; 17D99;

Key words and phrases. Evolution algebra, chain of evolution algebra, Kolmogorov-Chapman equation, isomorphism of algebras. 
field $\mathbb{R}$, with the basis $e_{1}, e_{2}, \ldots, e_{n}$ and the multiplication table

$$
e_{i} e_{i}=\sum_{j=1}^{n} a_{i, j}^{[s, t]} e_{j}, \quad i=1, \ldots, n ; \quad e_{i} e_{j}=0, \quad i \neq j .
$$

Here parameters $s, t$ are considered as time.

Denote by $\mathcal{M}^{[s, t]}=\left(a_{i, j}^{[s, t]}\right)_{i, j=1, \ldots n}$ the matrix of structural constants of $E^{[s, t]}$.

Definition 1. A family $\left\{E^{[s, t]}: s, t \in \mathbb{R}, 0 \leq s \leq t\right\}$ of $n$-dimensional evolution algebras over the field $\mathbb{R}$, is called a chain of evolution algebras (CEA) if the matrix $\mathcal{M}^{[s, t]}$ of structural constants satisfies the Chapman-Kolmogorov equation

$$
\mathcal{M}^{[s, t]}=\mathcal{M}^{[s, \tau]} \mathcal{M}^{[\tau, t]}, \text { for any } s<\tau<t .
$$

In this paper we study the following known CEAs (constructed in [6]):

$E_{i}^{[s, t]}$, which correspond to the $\mathcal{M}_{i}^{[s, t]}, i=1,2,3$ defined as:

$$
\mathcal{M}_{1}^{[s, t]}=\frac{h(t)}{2}\left(\begin{array}{ccc}
\frac{1}{h(s)}+f(s) & \frac{1}{h(s)}+f(s) & \frac{1}{h(s)}+f(s) \\
\frac{1}{h(s)}-g(s) & \frac{1}{h(s)}-g(s) & \frac{1}{h(s)}-g(s) \\
g(s)-f(s) & g(s)-f(s) & g(s)-f(s)
\end{array}\right)
$$

where $h, g$ and $f$ are arbitrary functions with $h(s) \neq 0$;

$$
\mathcal{M}_{2}^{[s, t]}=\frac{1}{2} \begin{cases}\left(\begin{array}{ccc}
1+\psi(s) & 1+\psi(s) & 1+\psi(s) \\
1-\varphi(s) & 1-\varphi(s) & 1-\varphi(s) \\
\varphi(s)-\psi(s) & \varphi(s)-\psi(s) & \varphi(s)-\psi(s)
\end{array}\right), \quad \text { if } s \leq t<a, \\
\left(\begin{array}{ccc}
0 & 0 & 0 \\
0 & 0 & 0 \\
0 & 0 & 0
\end{array}\right), & \text { if } t \geq a,\end{cases}
$$

where $a>0$ and $\varphi, \psi$ are arbitrary functions.

$$
\mathcal{M}_{3}^{[s, t]}=\theta(s)\left(\begin{array}{ccc}
\eta(t) & \vartheta(t) & \kappa(t) \\
\varphi_{1}(s) \eta(t) & \varphi_{1}(s) \vartheta(t) & \varphi_{1}(s) \kappa(t) \\
\varphi_{2}(s) \eta(t) & \varphi_{2}(s) \vartheta(t) & \varphi_{2}(s) \kappa(t)
\end{array}\right)
$$

where

$$
\theta(s)=\frac{1}{\eta(s)+\varphi_{1}(s) \vartheta(s)+\varphi_{2}(s) \kappa(s)}
$$

and $\eta, \vartheta, \kappa, \varphi_{1}, \varphi_{2}$ are arbitrary functions with $\eta(s)+\varphi_{1}(s) \vartheta(s)+\varphi_{2}(s) \kappa(s) \neq 0$.

In [7] (see also [1] for the complex field case) three-dimensional real evolution algebras with $\operatorname{dim}\left(E^{2}\right)=1$ are classified and twelve pairwise non-isomorphic evolution algebras are described. They are given by the following matrices of structural constants: 


$$
\begin{aligned}
& E_{1}:\left(\begin{array}{ccc}
1 & 1 & 0 \\
-1 & -1 & 0 \\
0 & 0 & 0
\end{array}\right), E_{2}:\left(\begin{array}{ccc}
1 & 1 & 0 \\
-1 & -1 & 0 \\
1 & 1 & 0
\end{array}\right), E_{3}:\left(\begin{array}{ccc}
1 & 1 & 0 \\
-1 & -1 & 0 \\
-1 & -1 & 0
\end{array}\right), E_{4}:\left(\begin{array}{lll}
1 & 0 & 0 \\
0 & 0 & 0 \\
0 & 0 & 0
\end{array}\right), \\
& E_{5}:\left(\begin{array}{ccc}
1 & 0 & 0 \\
0 & 0 & 0 \\
1 & 0 & 0
\end{array}\right), E_{6}:\left(\begin{array}{rrr}
1 & 0 & 0 \\
0 & 0 & 0 \\
-1 & 0 & 0
\end{array}\right), E_{7}:\left(\begin{array}{lll}
1 & 0 & 0 \\
1 & 0 & 0 \\
1 & 0 & 0
\end{array}\right), E_{8}:\left(\begin{array}{ccc}
1 & 0 & 0 \\
1 & 0 & 0 \\
-1 & 0 & 0
\end{array}\right), \\
& E_{9}:\left(\begin{array}{ccc}
1 & 0 & 0 \\
-1 & 0 & 0 \\
-1 & 0 & 0
\end{array}\right), E_{10}:\left(\begin{array}{ccc}
0 & 0 & 0 \\
0 & 0 & 0 \\
1 & 0 & 0
\end{array}\right), E_{11}:\left(\begin{array}{ccc}
0 & 0 & 0 \\
1 & 0 & 0 \\
1 & 0 & 0
\end{array}\right), E_{12}:\left(\begin{array}{ccc}
0 & 0 & 0 \\
1 & 0 & 0 \\
-1 & 0 & 0
\end{array}\right) \text {. }
\end{aligned}
$$

The main problem of this paper: For each CEA $E_{i}^{[s, t]}, i=1,2,3$ (listed above), to classify all EAs involved in the CEA. That is for each algebra $E_{j}, j=1,2, \ldots, 12$ to describe the set of two-dimensional times $(s, t)$ for which $E_{i}^{[s, t]}$ is isomorphic to the algebra $E_{j}$.

\section{On Classification of Algebras In Chains $E_{i}^{[s, t]}, i=1,2,3$.}

To give classification of algebras in three-dimensional real chains of evolution algebras we shall prove some lemmas, which are important to prove the main theorems of this section.

2.1. The case $E_{1}^{[s, t]}$. Any structural constants matrix of $E_{1}^{[s, t]}$ has the form as in the following lemma.

Lemma 1. The real evolution algebra corresponding to the matrix

$$
\mathcal{M}=\left(\begin{array}{lll}
\lambda & \lambda & \lambda \\
\mu & \mu & \mu \\
\gamma & \gamma & \gamma
\end{array}\right) \text { with conditions } \lambda+\mu+\gamma \neq 0 \text { is isomorphic to one of the following }
$$
algebras:

(a) $E_{4}$ if one of the following conditions is hold:

1) $\lambda \neq 0, \mu=0, \gamma=0$

2) $\mu \neq 0, \lambda=0, \gamma=0$

3) $\gamma \neq 0, \lambda=0, \mu=0$;

(b) $E_{5}$ if one of the following conditions is hold:

1) $\lambda=0, \mu \gamma>0$

2) $\mu=0, \lambda \gamma>0$

3) $\gamma=0, \lambda \mu>0$;

(c) $E_{6}$ if one of the following conditions is hold:

1) $\lambda=0, \mu \gamma<0$;

2) $\mu=0, \lambda \gamma<0$;

3) $\gamma=0, \lambda \mu<0$ 
(d) $E_{7}$ if the following conditions is hold:

$\lambda \mu \gamma \neq 0, \lambda \mu(\lambda+\mu)(\lambda+\mu+\gamma)>0, \gamma(\lambda+\mu)>0$

(e) $E_{8}$ if one of the following conditions is hold:

1) $\lambda \mu \gamma \neq 0, \lambda \mu(\lambda+\mu)(\lambda+\mu+\gamma)>0, \gamma(\lambda+\mu)<0$;

2) $\lambda \mu \gamma \neq 0, \lambda \mu(\lambda+\mu)(\lambda+\mu+\gamma)<0, \gamma(\lambda+\mu)>0$;

3) $\lambda \mu \gamma \neq 0, \lambda+\mu=0$

(f) $E_{9}$ if the following conditions is hold:

$\lambda \mu \gamma \neq 0, \lambda \mu(\lambda+\mu)(\lambda+\mu+\gamma)<0, \gamma(\lambda+\mu)<0$.

Proof. Let $E_{\mathcal{M}}$ be the evolution algebra with basis $\left\{e_{1}, e_{2}, e_{3}\right\}$. Then, the multiplication table in $E_{\mathcal{M}}$ is

$$
\begin{aligned}
& e_{1} e_{1}=\lambda e_{1}+\lambda e_{2}+\lambda e_{3}, e_{2} e_{2}=\mu e_{1}+\mu e_{2}+\mu e_{3}, \\
& e_{3} e_{3}=\gamma e_{1}+\gamma e_{2}+\gamma e_{3}, e_{1} e_{2}=e_{1} e_{3}=e_{2} e_{3}=0 .
\end{aligned}
$$

Let us consider $E_{i}, i=1, \ldots, 12$ listed in previous section. First we consider $E_{1}$, the multiplication table in $E_{1}$ is

$$
\begin{gathered}
e_{1}^{\prime} e_{1}^{\prime}=e_{1}^{\prime}+e_{2}^{\prime}, e_{2}^{\prime} e_{2}^{\prime}=-e_{1}^{\prime}-e_{2}^{\prime}, e_{3}^{\prime} e_{3}^{\prime}=0, \\
e_{1}^{\prime} e_{2}^{\prime}=0, e_{1}^{\prime} e_{3}^{\prime}=0, e_{2}^{\prime} e_{3}^{\prime}=0 .
\end{gathered}
$$

Assume that $E_{\mathcal{M}}$ is isomorphic to the algebra $E_{1}$. Then there exists a change of basis as follows

$$
\begin{gathered}
e_{1}^{\prime}=x_{1} e_{1}+x_{2} e_{2}+x_{3} e_{3}, \\
e_{2}^{\prime}=y_{1} e_{1}+y_{2} e_{2}+y_{3} e_{3}, \\
e_{3}^{\prime}=z_{1} e_{1}+z_{2} e_{2}+z_{3} e_{3}
\end{gathered}
$$

where determinant of the change is non-zero, i.e.,

$$
x_{1} y_{2} z_{3}+x_{3} y_{1} z_{2}+x_{2} y_{3} z_{1} \neq x_{3} y_{2} z_{1}+x_{2} y_{1} z_{3}+x_{1} y_{3} z_{2} .
$$

From the equalities (2.1) and (2.3) we obtain the following equations:

$$
\begin{gathered}
e_{1}^{\prime} e_{1}^{\prime}=\left(x_{1} e_{1}+x_{2} e_{2}+x_{3} e_{3}\right)\left(x_{1} e_{1}+x_{2} e_{2}+x_{3} e_{3}\right)=\left(\lambda x_{1}^{2}+\mu x_{2}^{2}+\gamma x_{3}^{2}\right)\left(e_{1}+e_{2}+e_{3}\right), \\
e_{2}^{\prime} e_{2}^{\prime}=\left(y_{1} e_{1}+y_{2} e_{2}+y_{3} e_{3}\right)\left(y_{1} e_{1}+y_{2} e_{2}+y_{3} e_{3}\right)=\left(\lambda y_{1}^{2}+\mu y_{2}^{2}+\gamma y_{3}^{2}\right)\left(e_{1}+e_{2}+e_{3}\right), \\
e_{3}^{\prime} e_{3}^{\prime}=\left(z_{1} e_{1}+z_{2} e_{2}+z_{3} e_{3}\right)\left(z_{1} e_{1}+z_{2} e_{2}+z_{3} e_{3}\right)=\left(\lambda z_{1}^{2}+\mu z_{2}^{2}+\gamma z_{3}^{2}\right)\left(e_{1}+e_{2}+e_{3}\right), \\
e_{1}^{\prime} e_{2}^{\prime}=\left(x_{1} e_{1}+x_{2} e_{2}+x_{3} e_{3}\right)\left(y_{1} e_{1}+y_{2} e_{2}+y_{3} e_{3}\right)=\left(\lambda x_{1} y_{1}+\mu x_{2} y_{2}+\gamma x_{3} y_{3}\right)\left(e_{1}+e_{2}+e_{3}\right), \\
e_{1}^{\prime} e_{3}^{\prime}=\left(x_{1} e_{1}+x_{2} e_{2}+x_{3} e_{3}\right)\left(z_{1} e_{1}+z_{2} e_{2}+z_{3} e_{3}\right)=\left(\lambda x_{1} z_{1}+\mu x_{2} z_{2}+\gamma x_{3} z_{3}\right)\left(e_{1}+e_{2}+e_{3}\right), \\
e_{2}^{\prime} e_{3}^{\prime}=\left(y_{1} e_{1}+y_{2} e_{2}+y_{3} e_{3}\right)\left(z_{1} e_{1}+z_{2} e_{2}+z_{3} e_{3}\right)=\left(\lambda y_{1} z_{1}+\mu y_{2} z_{2}+\gamma y_{3} z_{3}\right)\left(e_{1}+e_{2}+e_{3}\right), \\
e_{1}^{\prime}+e_{2}^{\prime}=\left(x_{1}+y_{1}\right) e_{1}+\left(x_{2}+y_{2}\right) e_{2}+\left(x_{3}+y_{3}\right) e_{3}, \\
-e_{1}^{\prime}-e_{2}^{\prime}=\left(-x_{1}-y_{1}\right) e_{1}+\left(-x_{2}-y_{2}\right) e_{2}+\left(-x_{3}-y_{3}\right) e_{3} .
\end{gathered}
$$


Consequently, being $\left\{e_{1}^{\prime}, e_{2}^{\prime}, e_{3}^{\prime}\right\}$ is the basis of evolution algebra and from the equalities (2.2) we have

$$
\left\{\begin{array}{l}
\lambda x_{1}^{2}+\mu x_{2}^{2}+\gamma x_{3}^{2}=x_{1}+y_{1}=x_{2}+y_{2}=x_{3}+y_{3} \\
\lambda y_{1}^{2}+\mu y_{2}^{2}+\gamma y_{3}^{2}=-x_{1}-y_{1}=-x_{2}-y_{2}=-x_{3}-y_{3} \\
\lambda z_{1}^{2}+\mu z_{2}^{2}+\gamma z_{3}^{2}=0 \\
\lambda x_{1} y_{1}+\mu x_{2} y_{2}+\gamma x_{3} y_{3}=0 \\
\lambda x_{1} z_{1}+\mu x_{2} z_{2}+\gamma x_{3} z_{3}=0 \\
\lambda y_{1} z_{1}+\mu y_{2} z_{2}+\gamma y_{3} z_{3}=0 .
\end{array}\right.
$$

If we add the right and left sides of the first and second equations of the system (2.5), respectively, we obtain the following equation

$$
\lambda\left(x_{1}^{2}+y_{1}^{2}\right)+\mu\left(x_{2}^{2}+y_{2}^{2}\right)+\gamma\left(x_{3}^{2}+y_{3}^{2}\right)=0 .
$$

From this equation we get

$$
\begin{aligned}
& \lambda\left(x_{1}+y_{1}\right)^{2}-2 \lambda x_{1} y_{1}+\mu\left(x_{2}+y_{2}\right)^{2}-2 \mu x_{2} y_{2}+\gamma\left(x_{3}+y_{3}\right)^{2}-2 \gamma x_{3} y_{3}=0 \\
& \lambda\left(x_{1}+y_{1}\right)^{2}+\mu\left(x_{2}+y_{2}\right)^{2}+\gamma\left(x_{3}+y_{3}\right)^{2}-2\left(\lambda x_{1} y_{1}+\mu x_{2} y_{2}+\gamma x_{3} y_{3}\right)=0 .
\end{aligned}
$$

Denote $x_{1}+y_{1}=x_{2}+y_{2}=x_{3}+y_{3}=k$. If we use the fourth equation of the system (2.5), then this equation will be

$$
\lambda k^{2}+\mu k^{2}+\gamma k^{2}=0, k^{2}(\lambda+\mu+\gamma)=0, k^{2}(\lambda+\mu+\gamma)=0 .
$$

By the condition of the lemma $\lambda+\mu+\gamma \neq 0$, then $k=0$. It means that

$$
x_{1}+y_{1}=x_{2}+y_{2}=x_{3}+y_{3}=0, x_{1}=-y_{1}, x_{2}=-y_{2}, x_{3}=-y_{3} .
$$

It is a contradiction to 2.4, hence $E_{\mathcal{M}}$ is not isomorphic to the algebra $E_{1}$.

Now, let us consider the algebras $E_{2}$ and $E_{3}$. For these algebras systems of equations similar to (2.5) are as follows, respectively

$$
\left\{\begin{array}{l}
\lambda x_{1}^{2}+\mu x_{2}^{2}+\gamma x_{3}^{2}=x_{1}+y_{1}=x_{2}+y_{2}=x_{3}+y_{3} \\
\lambda y_{1}^{2}+\mu y_{2}^{2}+\gamma y_{3}^{2}=-x_{1}-y_{1}=-x_{2}-y_{2}=-x_{3}-y_{3} \\
\lambda z_{1}^{2}+\mu z_{2}^{2}+\gamma z_{3}^{2}=x_{1}+y_{1}=x_{2}+y_{2}=x_{3}+y_{3} \\
\lambda x_{1} y_{1}+\mu x_{2} y_{2}+\gamma x_{3} y_{3}=0 \\
\lambda x_{1} z_{1}+\mu x_{2} z_{2}+\gamma x_{3} z_{3}=0 \\
\lambda y_{1} z_{1}+\mu y_{2} z_{2}+\gamma y_{3} z_{3}=0
\end{array}\right.
$$

and

$$
\left\{\begin{array}{l}
\lambda x_{1}^{2}+\mu x_{2}^{2}+\gamma x_{3}^{2}=x_{1}+y_{1}=x_{2}+y_{2}=x_{3}+y_{3} \\
\lambda y_{1}^{2}+\mu y_{2}^{2}+\gamma y_{3}^{2}=-x_{1}-y_{1}=-x_{2}-y_{2}=-x_{3}-y_{3} \\
\lambda z_{1}^{2}+\mu z_{2}^{2}+\gamma z_{3}^{2}=-x_{1}-y_{1}=-x_{2}-y_{2}=-x_{3}-y_{3} \\
\lambda x_{1} y_{1}+\mu x_{2} y_{2}+\gamma x_{3} y_{3}=0 \\
\lambda x_{1} z_{1}+\mu x_{2} z_{2}+\gamma x_{3} z_{3}=0 \\
\lambda y_{1} z_{1}+\mu y_{2} z_{2}+\gamma y_{3} z_{3}=0 .
\end{array}\right.
$$

Similar to the above, it is not difficult to show that $E_{\mathcal{M}}$ is not isomorphic to the algebras $E_{2}$ and $E_{3}$.

Let us consider the next cases.

Case 1. One of $\lambda, \mu, \gamma$ is not equal to zero. 
Case 1.1. Let $\lambda \neq 0, \mu=0, \gamma=0$. We consider the algebra $E_{4}$. The multiplication table in $E_{4}$ is

$$
\begin{aligned}
& e_{1}^{\prime} e_{1}^{\prime}=e_{1}^{\prime}, e_{2}^{\prime} e_{2}^{\prime}=0, e_{3}^{\prime} e_{3}^{\prime}=0 \\
& e_{1}^{\prime} e_{2}^{\prime}=0, e_{1}^{\prime} e_{3}^{\prime}=0, e_{2}^{\prime} e_{3}^{\prime}=0
\end{aligned}
$$

In this case the system of equations similar to 2.5 is as follows

$$
\left\{\begin{array}{l}
\lambda x_{1}^{2}=x_{1}=x_{2}=x_{3} \\
\lambda y_{1}^{2}=0 \\
\lambda z_{1}^{2}=0 \\
\lambda x_{1} y_{1}=0 \\
\lambda x_{1} z_{1}=0 \\
\lambda y_{1} z_{1}=0
\end{array}\right.
$$

If we solve the first equation of this system then there will be two solutions:

1) If $x_{1}=0$ then $x_{2}=x_{3}=0$. It is contradiction to (2.4).

2) If $x_{1}=\frac{1}{\lambda}$ then $x_{2}=x_{3}=\frac{1}{\lambda}$ and $y_{1}=z_{1}=0$. In this case the coefficients of the change of basis (2.3) are as follows $x_{1}=x_{2}=x_{3}=\frac{1}{\lambda}, y_{1}=z_{1}=0$ and $y_{2}, y_{3}, z_{2}, z_{3}$ are arbitrary real numbers such that satisfy the condition $y_{2} z_{3} \neq y_{3} z_{2}$.

Without loss of generality we can take $y_{2}=1, y_{3}=0, z_{2}=0, z_{3}=1$. So by the change of basis $e_{1}^{\prime}=\frac{1}{\lambda} e_{1}+\frac{1}{\lambda} e_{2}+\frac{1}{\lambda} e_{3}, e_{2}^{\prime}=e_{2}, e_{3}^{\prime}=e_{3}$ we can see that the algebras $E_{\mathcal{M}}$ and $E_{4}$ are isomorphic.

Case 1.2. If $\lambda=0, \gamma=0, \mu \neq 0$ then we take the following basis change $\left\{e_{2}, e_{1}, e_{3}\right\}$ and we have case similar to the Case 1.1.

Case 1.3. If $\lambda=0, \mu=0, \gamma \neq 0$ then we take the following basis change $\left\{e_{3}, e_{2}, e_{1}\right\}$ and we have case similar to the Case 1.1.

Case 2. Two of $\lambda, \mu, \gamma$ are not equal to zero.

Case 2.1. Let $\lambda=0, \mu \gamma>0$. We consider the algebra $E_{5}$. The multiplication table in $E_{5}$ is

$$
\begin{gathered}
e_{1}^{\prime} e_{1}^{\prime}=e_{1}^{\prime}, e_{2}^{\prime} e_{2}^{\prime}=0, e_{3}^{\prime} e_{3}^{\prime}=e_{1}^{\prime}, \\
e_{1}^{\prime} e_{2}^{\prime}=0, e_{1}^{\prime} e_{3}^{\prime}=0, e_{2}^{\prime} e_{3}^{\prime}=0 .
\end{gathered}
$$

In this case the system of equations similar to 2.5 is as follows

$$
\left\{\begin{array}{l}
\mu x_{2}^{2}+\gamma x_{3}^{2}=x_{1}=x_{2}=x_{3} \\
\mu y_{2}^{2}+\gamma y_{3}^{2}=0 \\
\mu z_{2}^{2}+\gamma z_{3}^{2}=x_{1}=x_{2}=x_{3} \\
\mu x_{2} y_{2}+\gamma x_{3} y_{3}=0 \\
\mu x_{2} z_{2}+\gamma x_{3} z_{3}=0 \\
\mu y_{2} z_{2}+\gamma y_{3} z_{3}=0
\end{array}\right.
$$

From the first equation of this system we have the following solutions:

1) $x_{1}=x_{2}=x_{3}=0$. It is contradiction to 2.4. 
2) $x_{1}=x_{2}=x_{3}=\frac{1}{\mu+\gamma}$. In this case from the remaining equations of 2.6 we obtain the following system

$$
\left\{\begin{array}{l}
\mu y_{2}^{2}+\gamma y_{3}^{2}=0 \\
\mu z_{2}^{2}+\gamma z_{3}^{2}=\frac{1}{\mu+\gamma} \\
\mu y_{2}+\gamma y_{3}=0 \\
\mu z_{2}+\gamma z_{3}=0 \\
\mu y_{2} z_{2}+\gamma y_{3} z_{3}=0
\end{array}\right.
$$

Solution of this system is $y_{2}=0, y_{3}=0, z_{2}= \pm \sqrt{\frac{\gamma}{\mu}} \frac{1}{\mu+\gamma}, z_{3}=\mp \sqrt{\frac{\mu}{\gamma}} \frac{1}{\mu+\gamma}$.

We may take the coefficients of the change of basis (2.3) as follows

$$
\begin{aligned}
& x_{1}=x_{2}=x_{3}=\frac{1}{\mu+\gamma}, y_{1}=\alpha, y_{2}=0, y_{3}=0, \\
& z_{1}=\beta, z_{2}= \pm \sqrt{\frac{\gamma}{\mu}} \frac{1}{\mu+\gamma}, z_{3}=\mp \sqrt{\frac{\mu}{\gamma}} \frac{1}{\mu+\gamma},
\end{aligned}
$$

where $\alpha \neq 0, \alpha, \beta \in \mathbb{R}$. So, we may take the change of basis

$$
e_{1}^{\prime}=\frac{1}{\mu+\gamma} e_{1}+\frac{1}{\mu+\gamma} e_{2}+\frac{1}{\mu+\gamma} e_{3}, e_{2}^{\prime}=\alpha e_{1}, e_{3}^{\prime}=\beta e_{1}+\sqrt{\frac{\gamma}{\mu}} \frac{1}{\mu+\gamma} e_{2}-\sqrt{\frac{\mu}{\gamma}} \frac{1}{\mu+\gamma} e_{3}
$$

and the determinant of the matrix of this change is equal to $D=\frac{\alpha}{(\mu+\gamma) \sqrt{\mu \gamma}} \neq 0$. By this change of basis we can see that $E_{\mathcal{M}}$ and $E_{5}$ are isomorphic.

Case 2.2. Let $\lambda=0, \mu \gamma<0$. By the condition of the lemma $\mu+\gamma \neq 0$. In these case we may take the change of basis

$e_{1}^{\prime}=\frac{1}{\mu+\gamma} e_{1}+\frac{1}{\mu+\gamma} e_{2}+\frac{1}{\mu+\gamma} e_{3}, e_{2}^{\prime}=\alpha e_{1}, e_{3}^{\prime}=\beta e_{1}+\sqrt{-\frac{\gamma}{\mu}} \frac{1}{\mu+\gamma} e_{2}-\sqrt{-\frac{\mu}{\gamma}} \frac{1}{\mu+\gamma} e_{3}$

and the determinant of the matrix of this change is equal to $D=\frac{\alpha}{(\mu+\gamma)^{2}}\left(\sqrt{-\frac{\gamma}{\mu}}+\sqrt{-\frac{\mu}{\gamma}}\right) \neq 0$. By this change of basis we can see that $E_{\mathcal{M}}$ is isomorphic to $E_{6}$.

For the cases

(i) $\mu=0, \lambda \gamma>0$ and $\mu=0, \lambda \gamma<0$;

(ii) $\gamma=0, \lambda \mu>0$ and $\gamma=0, \lambda \mu<0$,

respectively, if we take the changes of basis as follows $\left\{e_{2}, e_{1}, e_{3}\right\},\left\{e_{3}, e_{2}, e_{1}\right\}$ then we will have cases similar to the Case 2.1 and Case 2.2.

Case 3. None of $\lambda, \mu, \gamma$ is zero, i.e $\lambda \mu \gamma \neq 0$.

Case 3.1. Let

$$
\lambda \mu(\lambda+\mu)(\lambda+\mu+\gamma)>0, \gamma(\lambda+\mu)>0 .
$$

We consider the algebra $E_{7}$. The multiplication table in $E_{7}$ is

$$
\begin{gathered}
e_{1}^{\prime} e_{1}^{\prime}=e_{1}^{\prime}, e_{2}^{\prime} e_{2}^{\prime}=e_{1}^{\prime}, e_{3}^{\prime} e_{3}^{\prime}=e_{1}^{\prime}, \\
e_{1}^{\prime} e_{2}^{\prime}=0, e_{1}^{\prime} e_{3}^{\prime}=0, e_{2}^{\prime} e_{3}^{\prime}=0 .
\end{gathered}
$$


In this case the system of equations similar to 2.5 is as follows

$$
\left\{\begin{array}{l}
\lambda x_{1}^{2}+\mu x_{2}^{2}+\gamma x_{3}^{2}=x_{1}=x_{2}=x_{3} \\
\lambda y_{1}^{2}+\mu y_{2}^{2}+\gamma y_{3}^{2}=x_{1}=x_{2}=x_{3} \\
\lambda z_{1}^{2}+\mu z_{2}^{2}+\gamma z_{3}^{2}=x_{1}=x_{2}=x_{3} \\
\lambda x_{1} y_{1}+\mu x_{2} y_{2}+\gamma x_{3} y_{3}=0 \\
\lambda x_{1} z_{1}+\mu x_{2} z_{2}+\gamma x_{3} z_{3}=0 \\
\lambda y_{1} z_{1}+\mu y_{2} z_{2}+\gamma y_{3} z_{3}=0 .
\end{array}\right.
$$

If we solve the first equation of this system then:

1) $x_{1}=x_{2}=x_{3}=0$. It is contradiction to (2.4).

2) $x_{1}=x_{2}=x_{3}=\frac{1}{\lambda+\mu+\gamma}$. In this case from the remaining equations of 2.8 we obtain the following system

$$
\left\{\begin{array}{l}
\lambda y_{1}^{2}+\mu y_{2}^{2}+\gamma y_{3}^{2}=\frac{1}{\lambda+\mu+\gamma} \\
\lambda z_{1}^{2}+\mu z_{2}^{2}+\gamma z_{3}^{2}=\frac{1}{\lambda+\mu+\gamma} \\
\lambda y_{1}+\mu y_{2}+\gamma y_{3}=0 \\
\lambda z_{1}+\mu z_{2}+\gamma z_{3}=0 \\
\lambda y_{1} z_{1}+\mu y_{2} z_{2}+\gamma y_{3} z_{3}=0
\end{array}\right.
$$

Without loss of generality we solve the system $(2.9)$ for the case $y_{3}=0$. Then we have

$$
\left\{\begin{array}{l}
\lambda y_{1}^{2}+\mu y_{2}^{2}=\frac{1}{\lambda+\mu+\gamma} \\
\lambda y_{1}+\mu y_{2}=0 \\
\lambda z_{1}^{2}+\mu z_{2}^{2}+\gamma z_{3}^{2}=\frac{1}{\lambda+\mu+\gamma} \\
\lambda z_{1}+\mu z_{2}+\gamma z_{3}=0 \\
\lambda y_{1} z_{1}+\mu y_{2} z_{2}=0
\end{array}\right.
$$

From the second equation of the system 2.10 we find $y_{1}=-\frac{\mu}{\lambda} y_{2}$ and put it in the first equation of this system then we have the next equation

$$
\frac{\mu^{2}}{\lambda} y_{2}^{2}+\mu y_{2}^{2}=\frac{1}{\lambda+\mu+\gamma}
$$

The solution of this equation is $y_{2}= \pm \sqrt{\frac{\lambda}{\mu(\lambda+\mu)(\lambda+\mu+\gamma)}}$. Note that, if the condition $\sqrt{2.7}$ is hold then $\lambda \mu>0$. Thus $y_{1}=\mp \sqrt{\frac{\mu}{\lambda(\lambda+\mu)(\lambda+\mu+\gamma)}}$.

So, we may take $y_{1}=-\sqrt{\frac{\mu}{\lambda(\lambda+\mu)(\lambda+\mu+\gamma)}}$ and $y_{2}=\sqrt{\frac{\lambda}{\mu(\lambda+\mu)(\lambda+\mu+\gamma)}}$. From the last equation of 2.10 we have

$$
-\lambda \sqrt{\frac{\mu}{\lambda(\lambda+\mu)(\lambda+\mu+\gamma)}} z_{1}+\mu \sqrt{\frac{\lambda}{\mu(\lambda+\mu)(\lambda+\mu+\gamma)}} z_{2}=0 .
$$

It means that $z_{1}=z_{2}$. From this equality and from the third and fourth equations of the system (2.10) we have the next system of equations

$$
\left\{\begin{array}{l}
\lambda z_{1}^{2}+\mu z_{1}^{2}+\gamma z_{3}^{2}=\frac{1}{\lambda+\mu+\gamma} \\
\lambda z_{1}+\mu z_{1}+\gamma z_{3}=0
\end{array}\right.
$$


Solution of this system is $z_{1}= \pm \sqrt{\frac{\gamma}{\lambda+\mu}} \frac{1}{\lambda+\mu+\gamma}, z_{3}=\mp \sqrt{\frac{\lambda+\mu}{\gamma}} \frac{1}{\lambda+\mu+\gamma}$.

Thus, one of the solution of the system $(2.8)$ is

$$
\begin{gathered}
x_{1}=x_{2}=x_{3}=\frac{1}{\lambda+\mu+\gamma}, y_{1}=-\sqrt{\frac{\mu}{\lambda(\lambda+\mu)(\lambda+\mu+\gamma)}}, y_{2}=\sqrt{\frac{\lambda}{\mu(\lambda+\mu)(\lambda+\mu+\gamma)}}, y_{3}=0, \\
z_{1}=z_{2}=\sqrt{\frac{\gamma}{\lambda+\mu}} \frac{1}{\lambda+\mu+\gamma}, z_{3}=-\sqrt{\frac{\lambda+\mu}{\gamma}} \frac{1}{\lambda+\mu+\gamma} .
\end{gathered}
$$

Consequently, if we take the following change of basis

$$
\begin{gathered}
e_{1}^{\prime}=\frac{1}{\lambda+\mu+\gamma} e_{1}+\frac{1}{\lambda+\mu+\gamma} e_{2}+\frac{1}{\lambda+\mu+\gamma} e_{3}, \\
e_{2}^{\prime}=-\sqrt{\frac{\mu}{\lambda(\lambda+\mu)(\lambda+\mu+\gamma)}} e_{1}+\sqrt{\frac{\lambda}{\mu(\lambda+\mu)(\lambda+\mu+\gamma)}} e_{2}, \\
e_{3}^{\prime}=\sqrt{\frac{\gamma}{\lambda+\mu}} \frac{1}{\lambda+\mu+\gamma} e_{1}+\sqrt{\frac{\gamma}{\lambda+\mu}} \frac{1}{\lambda+\mu+\gamma} e_{2}-\sqrt{\frac{\lambda+\mu}{\gamma}} \frac{1}{\lambda+\mu+\gamma} e_{3}
\end{gathered}
$$

then the algebra $E_{\mathcal{M}}$ will be isomorphic to $E_{7}$. Indeed, according to the (2.1)

$$
\begin{gathered}
e_{1}^{\prime} e_{1}^{\prime}=\frac{1}{(\lambda+\mu+\gamma)^{2}} e_{1}^{2}+\frac{1}{(\lambda+\mu+\gamma)^{2}} e_{2}^{2}+\frac{1}{(\lambda+\mu+\gamma)^{2}} e_{3}^{2}=\frac{e_{1}+e_{2}+e_{3}}{\lambda+\mu+\gamma}=e_{1}^{\prime}, \\
e_{2}^{\prime} e_{2}^{\prime}=\frac{\mu}{\lambda(\lambda+\mu)(\lambda+\mu+\gamma)} e_{1}^{2}+\frac{\lambda}{\mu(\lambda+\mu)(\lambda+\mu+\gamma)} e_{2}^{2}=\frac{\mu\left(e_{1}+e_{2}+e_{3}\right)}{(\lambda+\mu)(\lambda+\mu+\gamma)}+\frac{\lambda\left(e_{1}+e_{2}+e_{3}\right)}{(\lambda+\mu)(\lambda+\mu+\gamma)}=e_{1}^{\prime}, \\
e_{3}^{\prime} e_{3}^{\prime}=\frac{\gamma}{\lambda+\mu} \frac{e_{1}^{2}}{(\lambda+\mu+\gamma)^{2}}+\frac{\gamma}{\lambda+\mu} \frac{e_{2}^{2}}{(\lambda+\mu+\gamma)^{2}}+\frac{\lambda+\mu}{\gamma} \frac{e_{3}^{2}}{(\lambda+\mu+\gamma)^{2}}=\frac{\lambda \gamma}{(\lambda+\mu)(\lambda+\mu+\gamma)^{2}}\left(e_{1}+e_{2}+e_{3}\right)+ \\
+\frac{\mu \gamma}{(\lambda+\mu)(\lambda+\mu+\gamma)^{2}}\left(e_{1}+e_{2}+e_{3}\right)+\frac{\lambda+\mu}{(\lambda+\mu+\gamma)^{2}}\left(e_{1}+e_{2}+e_{3}\right)=\frac{e_{1}+e_{2}+e_{3}}{\lambda+\mu+\gamma}=e_{1}^{\prime}
\end{gathered}
$$

and $e_{1}^{\prime} e_{2}^{\prime}=0, e_{1}^{\prime} e_{3}^{\prime}=0, e_{2}^{\prime} e_{3}^{\prime}=0$.

The determinant of the matrix of the change 2.11 is $D=\frac{ \pm 1}{(\lambda+\mu+\gamma) \sqrt{(\lambda+\mu+\gamma) \lambda \mu \gamma}} \neq 0$.

Case 3.2. If

$$
\lambda \mu(\lambda+\mu)(\lambda+\mu+\gamma)>0, \gamma(\lambda+\mu)<0, \lambda \mu>0
$$

then similarly to the above, we may take the following change of basis

$$
\begin{gathered}
e_{1}^{\prime}=\frac{1}{\lambda+\mu+\gamma} e_{1}+\frac{1}{\lambda+\mu+\gamma} e_{2}+\frac{1}{\lambda+\mu+\gamma} e_{3} \\
e_{2}^{\prime}=-\sqrt{\frac{\mu}{\lambda(\lambda+\mu)(\lambda+\mu+\gamma)}} e_{1}+\sqrt{\frac{\lambda}{\mu(\lambda+\mu)(\lambda+\mu+\gamma)}} e_{2}, \\
e_{3}^{\prime}=\sqrt{-\frac{\gamma}{\lambda+\mu}} \frac{1}{\lambda+\mu+\gamma} e_{1}+\sqrt{-\frac{\gamma}{\lambda+\mu}} \frac{1}{\lambda+\mu+\gamma} e_{2}+\sqrt{-\frac{\lambda+\mu}{\gamma}} \frac{1}{\lambda+\mu+\gamma} e_{3} .
\end{gathered}
$$

The determinant of the matrix of the change 2.12 is not equal to zero and for this basis it is not difficult to check that

$$
\begin{gathered}
e_{1}^{\prime} e_{1}^{\prime}=e_{1}^{\prime}, e_{2}^{\prime} e_{2}^{\prime}=e_{1}^{\prime}, e_{3}^{\prime} e_{3}^{\prime}=-e_{1}^{\prime}, \\
e_{1}^{\prime} e_{2}^{\prime}=0, e_{1}^{\prime} e_{3}^{\prime}=0, e_{2}^{\prime} e_{3}^{\prime}=0
\end{gathered}
$$

It means that in this case the algebra $E_{\mathcal{M}}$ is isomorphic to $E_{8}$.

\section{Case 3.3. If}

$$
\lambda \mu(\lambda+\mu)(\lambda+\mu+\gamma)>0, \gamma(\lambda+\mu)<0, \lambda \mu<0 .
$$


then we take the following change of basis

$$
\begin{gathered}
e_{1}^{\prime}=\frac{1}{\lambda+\mu+\gamma} e_{1}+\frac{1}{\lambda+\mu+\gamma} e_{2}+\frac{1}{\lambda+\mu+\gamma} e_{3}, \\
e_{2}^{\prime}=\sqrt{\frac{\mu}{\lambda(\lambda+\mu)(\lambda+\mu+\gamma)}} e_{1}+\sqrt{\frac{\lambda}{\mu(\lambda+\mu)(\lambda+\mu+\gamma)}} e_{2}, \\
e_{3}^{\prime}=\sqrt{-\frac{\gamma}{\lambda+\mu}} \frac{1}{\lambda+\mu+\gamma} e_{1}+\sqrt{-\frac{\gamma}{\lambda+\mu}} \frac{1}{\lambda+\mu+\gamma} e_{2}+\sqrt{-\frac{\lambda+\mu}{\gamma}} \frac{1}{\lambda+\mu+\gamma} e_{3} .
\end{gathered}
$$

By this change of basis we can see that the algebra $E_{\mathcal{M}}$ is isomorphic to $E_{8}$ again.

Case 3.4. Let

$$
\lambda \mu(\lambda+\mu)(\lambda+\mu+\gamma)<0, \gamma(\lambda+\mu)>0 .
$$

Note that, if the condition (2.13) is hold then $\lambda \mu<0$. Then similarly to the above, in this case the algebra $E_{\mathcal{M}}$ is isomorphic to $E_{8}$. For this we take the change of basis:

$$
\begin{gathered}
e_{1}^{\prime}=\frac{1}{\lambda+\mu+\gamma} e_{1}+\frac{1}{\lambda+\mu+\gamma} e_{2}+\frac{1}{\lambda+\mu+\gamma} e_{3}, \\
e_{2}^{\prime}=\sqrt{\frac{\gamma}{\lambda+\mu}} \frac{1}{\lambda+\mu+\gamma} e_{1}+\sqrt{\frac{\gamma}{\lambda+\mu}} \frac{1}{\lambda+\mu+\gamma} e_{2}-\sqrt{\frac{\lambda+\mu}{\gamma}} \frac{1}{\lambda+\mu+\gamma} e_{3}, \\
e_{3}^{\prime}=\sqrt{-\frac{\mu}{\lambda(\lambda+\mu)(\lambda+\mu+\gamma)}} e_{1}+\sqrt{-\frac{\lambda}{\mu(\lambda+\mu)(\lambda+\mu+\gamma)}} e_{2} .
\end{gathered}
$$

The determinant of the matrix of the change $(2.14)$ is not equal to zero and for this basis it is easy to check that

$$
\begin{gathered}
e_{1}^{\prime} e_{1}^{\prime}=e_{1}^{\prime}, e_{2}^{\prime} e_{2}^{\prime}=e_{1}^{\prime}, e_{3}^{\prime} e_{3}^{\prime}=-e_{1}^{\prime}, \\
e_{1}^{\prime} e_{2}^{\prime}=0, e_{1}^{\prime} e_{3}^{\prime}=0, e_{2}^{\prime} e_{3}^{\prime}=0 .
\end{gathered}
$$

\section{Case 3.5. If}

$$
\lambda \mu(\lambda+\mu)(\lambda+\mu+\gamma)<0, \gamma(\lambda+\mu)<0, \lambda \mu>0
$$

then we take the following change of basis

$$
\begin{gathered}
e_{1}^{\prime}=\frac{1}{\lambda+\mu+\gamma} e_{1}+\frac{1}{\lambda+\mu+\gamma} e_{2}+\frac{1}{\lambda+\mu+\gamma} e_{3}, \\
e_{2}^{\prime}=-\sqrt{-\frac{\mu}{\lambda(\lambda+\mu)(\lambda+\mu+\gamma)}} e_{1}+\sqrt{-\frac{\lambda}{\mu(\lambda+\mu)(\lambda+\mu+\gamma)}} e_{2}, \\
e_{3}^{\prime}=\sqrt{-\frac{\gamma}{\lambda+\mu}} \frac{1}{\lambda+\mu+\gamma} e_{1}+\sqrt{-\frac{\gamma}{\lambda+\mu}} \frac{1}{\lambda+\mu+\gamma} e_{2}+\sqrt{-\frac{\lambda+\mu}{\gamma}} \frac{1}{\lambda+\mu+\gamma} e_{3} .
\end{gathered}
$$

The determinant of the matrix of the change (2.15) is not equal to zero and for this basis it is not difficult to check that

$$
\begin{gathered}
e_{1}^{\prime} e_{1}^{\prime}=e_{1}^{\prime}, e_{2}^{\prime} e_{2}^{\prime}=-e_{1}^{\prime}, e_{3}^{\prime} e_{3}^{\prime}=-e_{1}^{\prime}, \\
e_{1}^{\prime} e_{2}^{\prime}=0, e_{1}^{\prime} e_{3}^{\prime}=0, e_{2}^{\prime} e_{3}^{\prime}=0 .
\end{gathered}
$$

It means that in this case the algebra $E_{\mathcal{M}}$ is isomorphic to $E_{9}$.

Case 3.6. If

$$
\lambda \mu(\lambda+\mu)(\lambda+\mu+\gamma)<0, \gamma(\lambda+\mu)<0, \lambda \mu<0
$$

then we take the following change of basis

$$
\begin{gathered}
e_{1}^{\prime}=\frac{1}{\lambda+\mu+\gamma} e_{1}+\frac{1}{\lambda+\mu+\gamma} e_{2}+\frac{1}{\lambda+\mu+\gamma} e_{3} \\
e_{2}^{\prime}=\sqrt{-\frac{\mu}{\lambda(\lambda+\mu)(\lambda+\mu+\gamma)}} e_{1}+\sqrt{-\frac{\lambda}{\mu(\lambda+\mu)(\lambda+\mu+\gamma)}} e_{2} \\
e_{3}^{\prime}=\sqrt{-\frac{\gamma}{\lambda+\mu}} \frac{1}{\lambda+\mu+\gamma} e_{1}+\sqrt{-\frac{\gamma}{\lambda+\mu}} \frac{1}{\lambda+\mu+\gamma} e_{2}+\sqrt{-\frac{\lambda+\mu}{\gamma}} \frac{1}{\lambda+\mu+\gamma} e_{3} .
\end{gathered}
$$


By this change of basis we can see that the algebra $E_{\mathcal{M}}$ is isomorphic to $E_{9}$ again.

Case 3.7. Let $\lambda+\mu=0$.

Case 3.7.1. If $\lambda(\lambda+\gamma)>0$ then we take the following change of basis

$$
\begin{gathered}
e_{1}^{\prime}=\frac{1}{\gamma} e_{1}+\frac{1}{\gamma} e_{2}+\frac{1}{\gamma} e_{3}, e_{2}^{\prime}=\frac{1}{\sqrt{\lambda(\lambda+\gamma)}} e_{1}-\frac{\lambda}{\gamma} \frac{1}{\sqrt{\lambda(\lambda+\gamma)}} e_{3}, \\
e_{3}^{\prime}=\frac{1}{\gamma} \sqrt{\frac{\lambda}{\lambda+\gamma}} e_{1}+\frac{\lambda+\gamma}{\lambda \gamma} \sqrt{\frac{\lambda}{\lambda+\gamma}} e_{2}+\frac{1}{\gamma} \sqrt{\frac{\lambda}{\lambda+\gamma}} e_{3} .
\end{gathered}
$$

By this change of basis we can see that the algebra $E_{\mathcal{M}}$ is isomorphic to $E_{8}$.

Case 3.7.2. If $\lambda(\lambda+\gamma)<0$ then we take the following change of basis

$$
\begin{gathered}
e_{1}^{\prime}=\frac{1}{\gamma} e_{1}+\frac{1}{\gamma} e_{2}+\frac{1}{\gamma} e_{3}, \\
e_{2}^{\prime}=\frac{1}{\gamma} \sqrt{\frac{-\lambda}{\lambda+\gamma}} e_{1}+\frac{\lambda+\gamma}{\lambda \gamma} \sqrt{\frac{-\lambda}{\lambda+\gamma}} e_{2}+\frac{1}{\gamma} \sqrt{\frac{-\lambda}{\lambda+\gamma}} e_{3}, \\
e_{3}^{\prime}=\frac{1}{\sqrt{-\lambda(\lambda+\gamma)}} e_{1}-\frac{\lambda}{\gamma} \frac{1}{\sqrt{-\lambda(\lambda+\gamma)}} e_{3} .
\end{gathered}
$$

By this change of basis we can see that the algebra $E_{\mathcal{M}}$ is isomorphic to $E_{8}$ again.

Case 3.7.3. If $\lambda+\gamma=0$ then we take the following change of basis

$$
\begin{gathered}
e_{1}^{\prime}=\frac{-1}{\lambda} e_{1}+\frac{-1}{\lambda} e_{2}+\frac{-1}{\lambda} e_{3}, e_{2}^{\prime}=\frac{1}{\sqrt{2} \lambda} e_{2}-\frac{1}{\sqrt{2} \lambda} e_{3}, \\
e_{3}^{\prime}=\frac{\sqrt{2}}{\lambda} e_{1}+\frac{1}{\sqrt{2} \lambda} e_{2}+\frac{1}{\sqrt{2} \lambda} e_{3} .
\end{gathered}
$$

In this case again the algebra $E_{\mathcal{M}}$ is isomorphic to the algebra $E_{8}$.

Now, we consider the algebra $E_{10}$. The multiplication table in $E_{10}$ is

$$
\begin{gathered}
e_{1}^{\prime} e_{1}^{\prime}=0, e_{2}^{\prime} e_{2}^{\prime}=0, e_{3}^{\prime} e_{3}^{\prime}=e_{1}^{\prime}, \\
e_{1}^{\prime} e_{2}^{\prime}=0, e_{1}^{\prime} e_{3}^{\prime}=0, e_{2}^{\prime} e_{3}^{\prime}=0 .
\end{gathered}
$$

Assume that $E_{\mathcal{M}}$ is isomorphic to the algebra $E_{10}$. Then there exists a change of basis as 2.3. In this case we have the following system of equations similar to 2.5

$$
\left\{\begin{array}{l}
\lambda x_{1}^{2}+\mu x_{2}^{2}+\gamma x_{3}^{2}=0 \\
\lambda y_{1}^{2}+\mu y_{2}^{2}+\gamma y_{3}^{2}=0 \\
\lambda z_{1}^{2}+\mu z_{2}^{2}+\gamma z_{3}^{2}=x_{1}=x_{2}=x_{3} \\
\lambda x_{1} y_{1}+\mu x_{2} y_{2}+\gamma x_{3} y_{3}=0 \\
\lambda x_{1} z_{1}+\mu x_{2} z_{2}+\gamma x_{3} z_{3}=0 \\
\lambda y_{1} z_{1}+\mu y_{2} z_{2}+\gamma y_{3} z_{3}=0
\end{array}\right.
$$

If we use $x_{1}=x_{2}=x_{3}$ and the first equation of (2.16) then we have the next equation

$$
x_{1}^{2}(\lambda+\mu+\gamma)=0 .
$$

We know that by the condition of the lemma $\lambda+\mu+\gamma \neq 0$. Hence $x_{1}=0$. It means that $x_{1}=x_{2}=x_{3}=0$. It is contradiction to (2.4), hence our assumption is not true.

In the same way, we can see that the algebra $E_{\mathcal{M}}$ is not isomorphic to the algebras $E_{11}$ and $E_{12}$.

Now, using this lemma we prove the following theorem which gives classification of the CEA: $E_{1}^{[s, t]}$, which correspond to the $\mathcal{M}_{1}^{[s, t]}$. 
Theorem 1. For given values $(s, t)$ of time the algebra $E_{1}^{[s, t]}$ is isomorphic to

(a) $E_{4}$ if one of the following conditions holds:

1) $g(s)=f(s)=\frac{1}{h(s)}$

2) $g(s)=f(s)=-\frac{1}{h(s)}$;

3) $g(s)=-f(s)=\frac{1}{h(s)}$;

(b) $E_{5}$ if one of the following conditions holds:

1) $f(s)=-\frac{1}{h(s)}, f^{2}(s)>g^{2}(s)$;

2) $g(s)=\frac{1}{h(s)}, g^{2}(s)>f^{2}(s)$;

3) $g(s)=f(s), \frac{1}{h^{2}(s)}>f^{2}(s)$;

(c) $E_{6}$ if one of the following conditions holds:

1) $f(s)=-\frac{1}{h(s)}, f^{2}(s)<g^{2}(s)$;

2) $g(s)=\frac{1}{h(s)}, g^{2}(s)<f^{2}(s)$;

3) $g(s)=f(s), \frac{1}{h^{2}(s)}<f^{2}(s)$;

(d) $E_{7}$ if the following conditions hold:

$\left(\frac{1}{h(s)}+f(s)\right)\left(\frac{1}{h(s)}-g(s)\right)(g(s)-f(s)) \neq 0,(g(s)-f(s))\left(\frac{2}{h(s)}+f(s)-g(s)\right)>0$ $\frac{2}{h(s)}\left(\frac{1}{h(s)}+f(s)\right)\left(\frac{1}{h(s)}-g(s)\right)\left(\frac{2}{h(s)}+f(s)-g(s)\right)>0 ;$

(e) $E_{8}$ if one of the following conditions holds:

1) $\left(\frac{1}{h(s)}+f(s)\right)\left(\frac{1}{h(s)}-g(s)\right)(g(s)-f(s)) \neq 0,(g(s)-f(s))\left(\frac{2}{h(s)}+f(s)-g(s)\right)<0$ $\frac{2}{h(s)}\left(\frac{1}{h(s)}+f(s)\right)\left(\frac{1}{h(s)}-g(s)\right)\left(\frac{2}{h(s)}+f(s)-g(s)\right)>0 ;$

2) $\left(\frac{1}{h(s)}+f(s)\right)\left(\frac{1}{h(s)}-g(s)\right)(g(s)-f(s)) \neq 0,(g(s)-f(s))\left(\frac{2}{h(s)}+f(s)-g(s)\right)>0$ $\frac{2}{h(s)}\left(\frac{1}{h(s)}+f(s)\right)\left(\frac{1}{h(s)}-g(s)\right)\left(\frac{2}{h(s)}+f(s)-g(s)\right)<0 ;$

3) $\left(\frac{1}{h(s)}+f(s)\right)\left(\frac{1}{h(s)}-g(s)\right)(g(s)-f(s)) \neq 0, \frac{2}{h(s)}+f(s)-g(s)=0$

(f) $E_{9}$ if the following conditions hold:

$$
\begin{aligned}
& \left(\frac{1}{h(s)}+f(s)\right)\left(\frac{1}{h(s)}-g(s)\right)(g(s)-f(s)) \neq 0,(g(s)-f(s))\left(\frac{2}{h(s)}+f(s)-g(s)\right)<0 \\
& \frac{2}{h(s)}\left(\frac{1}{h(s)}+f(s)\right)\left(\frac{1}{h(s)}-g(s)\right)\left(\frac{2}{h(s)}+f(s)-g(s)\right)<0 .
\end{aligned}
$$

Proof. We mention that in $\mathcal{M}_{1}^{[s, t]}$ the parameter functions $h, g$ and $f$ are arbitrary with $h(s) \neq 0$.

Let us denote

$\lambda(s, t)=\frac{1}{2} h(t)\left(\frac{1}{h(s)}+f(s)\right), \mu(s, t)=\frac{1}{2} h(t)\left(\frac{1}{h(s)}-g(s)\right), \gamma(s, t)=\frac{1}{2} h(t)(g(s)-f(s))$. 
Note that $\lambda(s, t)+\mu(s, t)+\gamma(s, t)=\frac{h(t)}{h(s)} \neq 0$.

a) If $g(s)=f(s)=\frac{1}{h(s)}$ then $\mu(s, t)=\gamma(s, t)=0$ and $\lambda(s, t)=\frac{h(t)}{h(s)} \neq 0$. By Lemma 1 , it is easy to see that $E_{1}^{[s, t]}$ is isomorphic to $E_{4}$.

The cases $g(s)=f(s)=-\frac{1}{h(s)}$ and $g(s)=-f(s)=\frac{1}{h(s)}$ are similarly.

b) Let us $f(s)=-\frac{1}{h(s)}, f^{2}(s)>g^{2}(s)$. It means that $\lambda(s, t)=0$ and $\mu(s, t) \gamma(s, t)>0$. Indeed

$$
\lambda(s, t)=\frac{1}{2} h(t)\left(\frac{1}{h(s)}+f(s)\right)=\frac{1}{2} h(t)\left(\frac{1}{h(s)}-\frac{1}{h(s)}\right)=0
$$

and

$$
\begin{gathered}
\mu(s, t) \gamma(s, t)=\frac{1}{4} h^{2}(t)\left(\frac{1}{h(s)}-g(s)\right)(g(s)-f(s))= \\
=\frac{1}{4} h^{2}(t)(-f(s)-g(s))(g(s)-f(s))=\frac{1}{4} h^{2}(t)\left(f^{2}(s)-g^{2}(s)\right)>0 .
\end{gathered}
$$

In this case by Lemma $1, E_{1}^{[s, t]}$ is isomorphic to $E_{5}$.

If the conditions $g(s)=\frac{1}{h(s)}, g^{2}(s)>f^{2}(s)$ and $g(s)=f(s), \frac{1}{h^{2}(s)}>f^{2}(s)$ are hold then $\mu(s, t)=0, \lambda(s, t) \gamma(s, t)>0$ and $\gamma(s, t)=0, \lambda(s, t) \mu(s, t)>0$ respectively. In these cases by Lemma $1, E_{1}^{[s, t]}$ is isomorphic to $E_{5}$ again.

c) Similar to the previous case, if $f(s)=-\frac{1}{h(s)}, f^{2}(s)<g^{2}(s)$ then $\lambda(s, t)=0$ and $\mu(s, t) \gamma(s, t)<0$. If the conditions $g(s)=\frac{1}{h(s)}, g^{2}(s)<f^{2}(s)$ and $g(s)=f(s), \frac{1}{h^{2}(s)}<$ $f^{2}(s)$ are hold then $\mu(s, t)=0, \lambda(s, t) \gamma(s, t)<0$ and $\gamma(s, t)=0, \lambda(s, t) \mu(s, t)<0$ respectively. In these cases $E_{1}^{[s, t]}$ is isomorphic to $E_{6}$.

d) If

$$
\begin{gathered}
\left(\frac{1}{h(s)}+f(s)\right)\left(\frac{1}{h(s)}-g(s)\right)(g(s)-f(s)) \neq 0,(g(s)-f(s))\left(\frac{2}{h(s)}+f(s)-g(s)\right)>0 \\
\frac{2}{h(s)}\left(\frac{1}{h(s)}+f(s)\right)\left(\frac{1}{h(s)}-g(s)\right)\left(\frac{2}{h(s)}+f(s)-g(s)\right)>0
\end{gathered}
$$

then

and

$$
\lambda(s, t) \mu(s, t) \gamma(s, t)=h^{3}(t)\left(\frac{1}{h(s)}+f(s)\right)\left(\frac{1}{h(s)}-g(s)\right)(g(s)-f(s)) \neq 0
$$

$$
\begin{gathered}
\gamma(s, t)(\lambda(s, t)+\mu(s, t))=\frac{1}{4} h^{2}(t)(g(s)-f(s))\left(\frac{2}{h(s)}+f(s)-g(s)\right)>0 \\
\lambda(s, t) \mu(s, t)(\lambda(s, t)+\mu(s, t))(\lambda(s, t)+\mu(s, t)+\gamma(s, t))= \\
=\frac{1}{8} \frac{h^{4}(t)}{h(s)}\left(\frac{1}{h(s)}+f(s)\right)\left(\frac{1}{h(s)}-g(s)\right)\left(\frac{2}{h(s)}+f(s)-g(s)\right)>0 .
\end{gathered}
$$

In this case by Lemma $1, E_{1}^{[s, t]}$ is isomorphic to $E_{7}$.

e) In the same way it is not difficult to see that if one of the following conditions is hold 
(i) $\left(\frac{1}{h(s)}+f(s)\right)\left(\frac{1}{h(s)}-g(s)\right)(g(s)-f(s)) \neq 0,(g(s)-f(s))\left(\frac{2}{h(s)}+f(s)-g(s)\right)<0$

$$
\frac{2}{h(s)}\left(\frac{1}{h(s)}+f(s)\right)\left(\frac{1}{h(s)}-g(s)\right)\left(\frac{2}{h(s)}+f(s)-g(s)\right)>0
$$

(ii) $\left(\frac{1}{h(s)}+f(s)\right)\left(\frac{1}{h(s)}-g(s)\right)(g(s)-f(s)) \neq 0,(g(s)-f(s))\left(\frac{2}{h(s)}+f(s)-g(s)\right)>0$

$$
\frac{2}{h(s)}\left(\frac{1}{h(s)}+f(s)\right)\left(\frac{1}{h(s)}-g(s)\right)\left(\frac{2}{h(s)}+f(s)-g(s)\right)<0
$$

(iii) $\left(\frac{1}{h(s)}+f(s)\right)\left(\frac{1}{h(s)}-g(s)\right)(g(s)-f(s)) \neq 0, \frac{2}{h(s)}+f(s)-g(s)=0$

then $E_{1}^{[s, t]}$ is isomorphic to $E_{8}$.

f) If

$$
\begin{gathered}
\left(\frac{1}{h(s)}+f(s)\right)\left(\frac{1}{h(s)}-g(s)\right)(g(s)-f(s)) \neq 0,(g(s)-f(s))\left(\frac{2}{h(s)}+f(s)-g(s)\right)<0 \\
\frac{1}{h(s)}\left(\frac{1}{h(s)}+f(s)\right)\left(\frac{1}{h(s)}-g(s)\right)\left(\frac{2}{h(s)}+f(s)-g(s)\right)<0
\end{gathered}
$$

then $E_{1}^{[s, t]}$ is isomorphic to $E_{9}$.

Remark 1. Note that by Lemma $1, E_{1}^{[s, t]}$ is not isomorphic to the algebras $E_{1}, E_{2}, E_{3}$, $E_{10}, E_{11}, E_{12}$ for any $0 \leq s \leq t$.

To illustrate the essence of Theorem 1, consider the following example.

Example 1. Let $g(s)=4 s-16, f(s)=4 s^{2}-24 s+32, \frac{1}{h(s)}=s+1$ then we obtain:
(a) $E_{4}$ when $s=3$;
(b) $E_{5}$ when $s=4$;
(c) $E_{6}$ when $s=\frac{11}{4}$ and $s=\frac{17}{3}$;
(d) $E_{7}$ when $s \in(3 ; 4)$;
(e) $E_{8}$ when $s \in\left[0 ; \frac{11}{4}\right) \cup\left(4 ; \frac{17}{3}\right)$;
(f) $E_{9}$ when $s \in\left(\frac{11}{4} ; 3\right) \cup\left(\frac{17}{3} ; \infty\right)$;

where $0 \leq s \leq t$ (see Fig. 1).

2.2. The case $E_{2}^{[s, t]}$. In this case by Lemma 1 we get

Lemma 2. The real evolution algebra corresponding to the matrix

$$
\left(\begin{array}{ccc}
1+a & 1+a & 1+a \\
1-b & 1-b & 1-b \\
b-a & b-a & b-a
\end{array}\right) \text { is isomorphic to one of the following algebras: }
$$

(a) $E_{4}$ if one of the following conditions is hold:

1) $a=b=1$;

2) $a=b=-1$;

3) $a=-1, b=1$;

(b) $E_{5}$ if one of the following conditions is hold: 


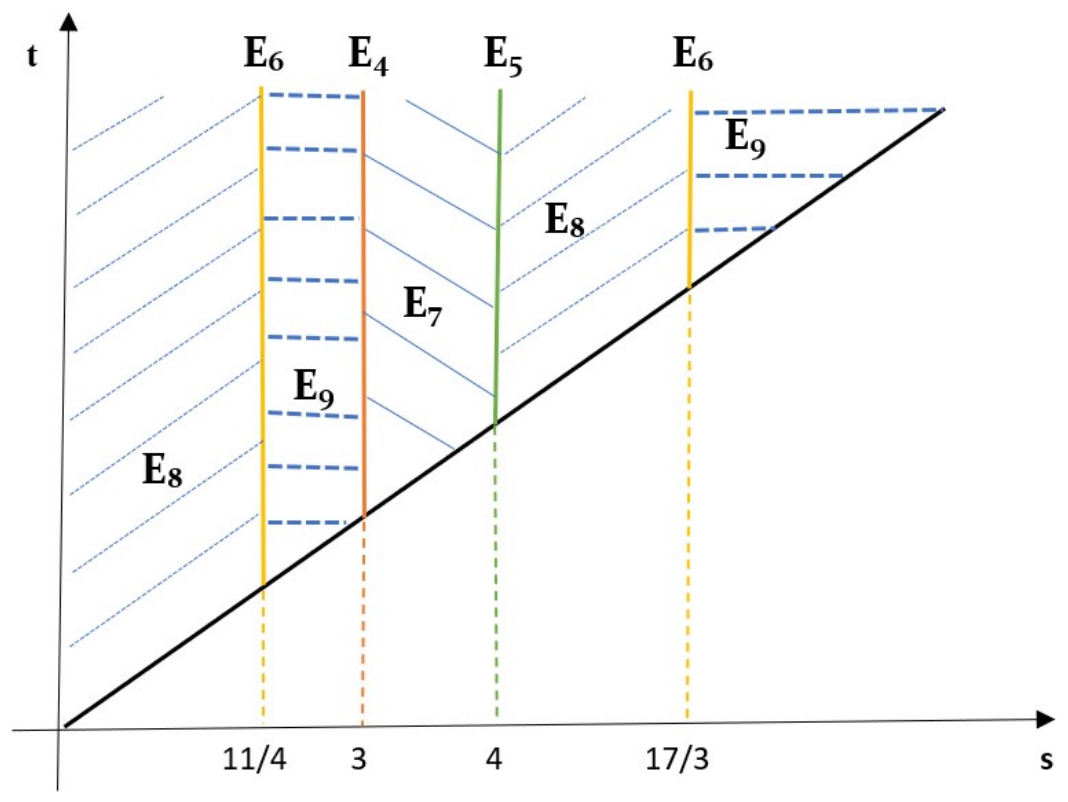

Figure 1. The partition of the set $\{(s, t): 0 \leq s \leq t\}$ corresponding to classification of EAs in the CEA $E_{1}^{[s, t]}$.

1) $a=-1,|b|<1$;

2) $b=1,|a|<1$;

3) $a=b,|a|<1$;

(c) $E_{6}$ if one of the following conditions is hold:

1) $a=-1,|b|>1$;

2) $b=1,|a|>1$;

3) $a=b,|a|>1$;

(d) $E_{7}$ if the following condition is hold:

$(1+a)(1-b)(b-a) \neq 0,(1+a)(1-b)(2+a-b)>0,(b-a)(2+a-b)>0 ;$

(e) $E_{8}$ if one of the following conditions is hold:

1) $(1+a)(1-b)(b-a) \neq 0,(1+a)(1-b)(2+a-b)>0,(b-a)(2+a-b)<0$;

2) $(1+a)(1-b)(b-a) \neq 0,(1+a)(1-b)(2+a-b)<0,(b-a)(2+a-b)>0$;

3) $(1+a)(1-b)(b-a) \neq 0,2+a-b=0$;

(f) $E_{9}$ if the following conditions is hold:

$(1+a)(1-b)(b-a) \neq 0,(1+a)(1-b)(2+a-b)<0,(b-a)(2+a-b)<0$. 
Now, by this lemma we obtain the following theorem which gives classification of the CEA: $E_{2}^{[s, t]}$. Let's denote by $E_{0}$ the trivial evolution algebra (i.e. with zero multiplication).

Theorem 2. For given values $(s, t) \in\{(s, t): 0 \leq s \leq t<a\}$ of time the algebra $E_{2}^{[s, t]}$ is isomorphic to

(a) $E_{4}$ if one of the following conditions is hold:

1) $\psi(s)=\varphi(s)=1$;

2) $\psi(s)=\varphi(s)=-1$;

3) $\psi(s)=-1, \varphi(s)=1$;

(b) $E_{5}$ if one of the following conditions is hold:

1) $\psi(s)=-1,|\varphi(s)|<1$;

2) $\varphi(s)=1,|\psi(s)|<1$

3) $\psi(s)=\varphi(s),|\psi(s)|<1$;

(c) $E_{6}$ if one of the following conditions is hold:

1) $\psi(s)=-1,|\varphi(s)|>1$;

2) $\varphi(s)=1,|\psi(s)|>1$;

3) $\psi(s)=\varphi(s),|\psi(s)|>1$;

(d) $E_{7}$ if the following condition is hold:

$(1+\psi(s))(1-\varphi(s))(\varphi(s)-\psi(s)) \neq 0$,

$(1+\psi(s))(1-\varphi(s))(2+\psi(s)-\varphi(s))>0,(\varphi(s)-\psi(s))(2+\psi(s)-\varphi(s))>0 ;$

(e) $E_{8}$ if one of the following conditions is hold:

1) $(1+\psi(s))(1-\varphi(s))(\varphi(s)-\psi(s)) \neq 0$,

$(1+\psi(s))(1-\varphi(s))(2+\psi(s)-\varphi(s))>0,(\varphi(s)-\psi(s))(2+\psi(s)-\varphi(s))<0 ;$

2) $(1+\psi(s))(1-\varphi(s))(\varphi(s)-\psi(s)) \neq 0$,

$(1+\psi(s))(1-\varphi(s))(2+\psi(s)-\varphi(s))<0,(\varphi(s)-\psi(s))(2+\psi(s)-\varphi(s))>0$

3) $(1+\psi(s))(1-\varphi(s))(\varphi(s)-\psi(s)) \neq 0,2+\psi(s)-\varphi(s)=0$;

(f) $E_{9}$ if the following condition is hold:

1) $(1+\psi(s))(1-\varphi(s))(\varphi(s)-\psi(s)) \neq 0$,

$(1+\psi(s))(1-\varphi(s))(2+\psi(s)-\varphi(s))<0,(\varphi(s)-\psi(s))(2+\psi(s)-\varphi(s))<0$.

For all $(s, t) \in\{(s, t): t \geq a\}$ the algebra $E_{2}^{[s, t]}$ is isomorphic to $E_{0}$.

Proof. The proof of the theorem is similar to that of Theorem 1.

Remark 2. By Lemma 2, $E_{2}^{[s, t]}$ is not isomorphic to the algebras $E_{1}, E_{2}, E_{3}, E_{10}, E_{11}$, $E_{12}$ for any $0 \leq s \leq t$.

To illustrate the essence of Theorem 2, consider the following example.

Example 2. Let $\varphi(s)=s^{2}-8 s+13, \psi(s)=s^{2}-5$ then for given 
$(s, t) \in\{(s, t): 0 \leq s \leq t<a\}$ we obtain:

(a) $E_{4}$ when $s=2$;

(b) $E_{5}$ when $s=\frac{9}{4}$;

(c) $E_{6}$ when $s=6$;

(d) $E_{7}$ when $s \in\left(2 ; \frac{9}{4}\right)$;

(e) $E_{8}$ when $s \in\left(\frac{9}{4} ; 6\right)$;

(f) $E_{9}$ when $s \in[0 ; 2) \cup(6 ; \infty)$;

and for all $(s, t) \in\{(s, t): t \geq a\}$ we obtain $E_{0}$ (see Fig. 2).

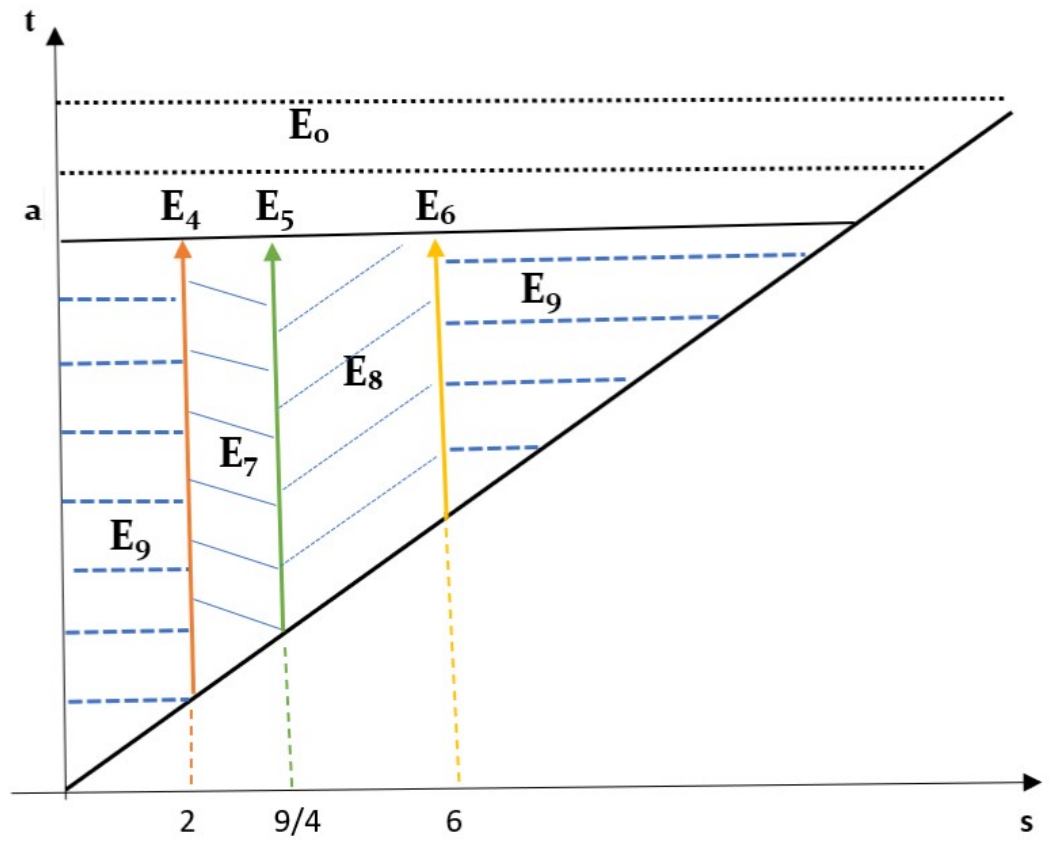

Figure 2. The partition of time set corresponding to the classification of the CEA $E_{2}^{[s, t]}$

Since the two different chains we considered above contain EAs isomorphic to the same algebras. But they do not include all twelve algebras $E_{i} i=1,2, \ldots, 12$. Below we show that $E_{3}^{[s, t]}$ is reach enough, one can choose its parameter functions such that the chain includes all the algebras.

\subsection{The case $E_{3}^{[s, t]}$.}

Lemma 3. For the real evolution algebra corresponding to the matrix 


$$
\left(\begin{array}{ccc}
\alpha & \beta & \gamma \\
\lambda \alpha & \lambda \beta & \lambda \gamma \\
\mu \alpha & \mu \beta & \mu \gamma
\end{array}\right) \text { we have }
$$

I. If $\alpha \neq 0, \alpha^{2}+\lambda \beta^{2}+\mu \gamma^{2}=0$ then it is isomorphic to one of the following algebras:

(a) $E_{1}$ if one of the following conditions is hold:

1) $\mu=0, \lambda \beta \gamma \neq 0$;

2) $\lambda=0, \beta \gamma \mu \neq 0$;

3) $\gamma=\mu=0, \lambda \beta \neq 0$;

4) $\lambda=\beta=0, \gamma \mu \neq 0$;

(b) $E_{2}$ if one of the following conditions is hold:

1) $\gamma=0, \lambda \beta \mu \neq 0, \mu>0$;

2) $\beta=0, \lambda \gamma \mu \neq 0, \lambda>0$;

3) $\lambda \beta \gamma \mu \neq 0$;

(c) $E_{3}$ if one of the following conditions is hold:

1) $\gamma=0, \lambda \beta \mu \neq 0, \mu<0$;

2) $\beta=0, \lambda \gamma \mu \neq 0, \lambda<0$.

II. If $\alpha \neq 0, \alpha^{2}+\lambda \beta^{2}+\mu \gamma^{2} \neq 0$ then it is isomorphic to one of the following algebras:

(d) $E_{4}$ if the following condition is hold:

$\lambda=0, \mu=0$;

(e) $E_{5}$ if one of the following conditions is hold:

1) $\lambda=0, \mu>0$;

2) $\mu=0, \lambda>0$

(f) $E_{6}$ if one of the following conditions is hold:

1) $\lambda=0, \mu<0$;

2) $\mu=0, \quad \lambda<0$;

(g) $E_{7}$ if the following condition is hold:

$\lambda>0, \mu>0$

(h) $E_{8}$ if one of the following conditions is hold:

1) $\lambda>0, \mu<0, \alpha^{2}+\lambda \beta^{2}+\mu \gamma^{2}>0$;

2) $\lambda<0, \mu>0, \alpha^{2}+\lambda \beta^{2}<0, \alpha^{2}+\lambda \beta^{2}+\mu \gamma^{2}>0$;

3) $\lambda<0, \mu>0, \alpha^{2}+\lambda \beta^{2}>0$;

4) $\lambda<0, \mu<0, \alpha^{2}+\lambda \beta^{2}>0, \alpha^{2}+\lambda \beta^{2}+\mu \gamma^{2}<0$;

5) $\lambda<0, \mu<0, \alpha^{2}+\lambda \beta^{2}<0$;

6) $\lambda \neq 0, \quad \mu \neq 0, \alpha^{2}+\lambda \beta^{2}=0$;

(i) $E_{9}$ if one of the following conditions is hold: 
1) $\lambda>0, \mu<0, \alpha^{2}+\lambda \beta^{2}+\mu \gamma^{2}<0$;

2) $\lambda<0, \mu>0, \alpha^{2}+\lambda \beta^{2}<0, \alpha^{2}+\lambda \beta^{2}+\mu \gamma^{2}<0$;

3) $\lambda<0, \mu<0, \alpha^{2}+\lambda \beta^{2}>0, \alpha^{2}+\lambda \beta^{2}+\mu \gamma^{2}>0$.

III. If $\alpha=0, \beta \neq 0$ then it is isomorphic to one of the following algebras:

(j) $E_{2}$ if the following condition is hold:

$\mu \neq 0, \gamma \neq 0, \lambda>0, \lambda \beta^{2}+\mu \gamma^{2}=0$

(k) $E_{3}$ if the following condition is hold:

$\mu \neq 0, \gamma \neq 0, \lambda<0, \lambda \beta^{2}+\mu \gamma^{2}=0$

(l) $E_{5}$ if one of the following conditions is hold:

1) $\mu=0, \lambda>0$

2) $\lambda=0, \gamma \neq 0, \mu>0$;

(m) $E_{6}$ if one of the following conditions is hold:

1) $\mu=0, \lambda<0$;

2) $\lambda=0, \gamma \neq 0, \mu<0$;

(n) $E_{7}$ if the following condition is hold:

$\lambda>0, \mu>0$;

(o) $E_{8}$ if one of the following conditions is hold:

1) $\lambda<0, \mu<0$;

2) $\lambda \beta^{2}+\mu \gamma^{2}>0, \lambda \mu<0$;

(p) $E_{9}$ if the following condition is hold:

$\lambda \beta^{2}+\mu \gamma^{2}<0, \lambda \mu<0$

(q) $E_{10}$ if the following condition is hold:

$\lambda=0, \mu=0$

(r) $E_{11}$ if the following condition is hold:

$\lambda=0, \gamma=0, \mu>0$;

(s) $E_{12}$ if the following condition is hold:

$\lambda=0, \gamma=0, \mu<0$;

Proof. Let

$$
M_{B}=\left(\begin{array}{ccc}
\alpha & \beta & \gamma \\
\lambda \alpha & \lambda \beta & \lambda \gamma \\
\mu \alpha & \mu \beta & \mu \gamma
\end{array}\right)
$$


be the matrix of structural constants of the evolution algebra $E_{M_{B}}$ relative to a basis $B=\left\{e_{1}, e_{2}, e_{3}\right\}$. Then, the multiplication table in $E_{M_{B}}$ is

$$
\begin{aligned}
& e_{1} e_{1}=\alpha e_{1}+\beta e_{2}+\gamma e_{3}, e_{2} e_{2}=\lambda \alpha e_{1}+\lambda \beta e_{2}+\lambda \gamma e_{3}, \\
& e_{3} e_{3}=\mu \alpha e_{1}+\mu \beta e_{2}+\mu \gamma e_{3}, e_{1} e_{2}=e_{1} e_{3}=e_{2} e_{3}=0 .
\end{aligned}
$$

We consider all possible cases.

Case 1. Suppose that $\alpha \neq 0$.

Case 1.1. Assume that $\alpha^{2}+\beta^{2} \lambda+\gamma^{2} \mu=0$. In this case may be one of the following cases:

1) $\mu=0, \lambda \beta \gamma \neq 0$

2) $\lambda=0, \beta \gamma \mu \neq 0$

3) $\gamma=0, \lambda \beta \mu \neq 0$;

4) $\beta=0, \lambda \gamma \mu \neq 0$

5) $\gamma=\mu=0, \lambda \beta \neq 0$;

6) $\lambda=\beta=0, \gamma \mu \neq 0$;

7) $\lambda \beta \gamma \mu \neq 0$.

Case 1.1.1. If $\mu=0, \lambda \beta \gamma \neq 0$ then $\alpha^{2}+\beta^{2} \lambda=0$. This implies that $\lambda=-\frac{\alpha^{2}}{\beta^{2}}$. In this case the matrix $M_{B}$ will be

$$
M_{B}=\left(\begin{array}{ccc}
\alpha & \beta & \gamma \\
-\frac{\alpha^{3}}{\beta^{2}} & -\frac{\alpha^{2}}{\beta} & -\frac{\gamma \alpha^{2}}{\beta^{2}} \\
0 & 0 & 0
\end{array}\right) .
$$

If we take the change of basis $B^{\prime}=\left\{\frac{1}{\alpha} e_{1}, \frac{\beta}{\alpha^{2}} e_{2}, \frac{\gamma}{\alpha^{2}} e_{3}\right\}$ then

$$
M_{B^{\prime}}=\left(\begin{array}{ccc}
1 & 1 & 1 \\
-1 & -1 & -1 \\
0 & 0 & 0
\end{array}\right) \text {. }
$$

Let take another change of basis $B^{\prime \prime}=\left\{-e_{2},-e_{1}-e_{3}, e_{3}\right\}$ and we find a structure matrix with more zeros. Then

$$
M_{B^{\prime \prime}}=\left(\begin{array}{ccc}
1 & 1 & 0 \\
-1 & -1 & 0 \\
0 & 0 & 0
\end{array}\right) .
$$

Consequently, in this case $E_{M_{B}}$ is isomorphic to $E_{1}$.

Case 1.1.2. Let $\lambda=0, \beta \gamma \mu \neq 0$ then $\alpha^{2}+\gamma^{2} \mu=0$. From this equality we find $\mu=-\frac{\alpha^{2}}{\gamma^{2}}$. In this case the structure matrix is:

$$
M_{B}=\left(\begin{array}{ccc}
\alpha & \beta & \gamma \\
0 & 0 & 0 \\
-\frac{\alpha^{3}}{\gamma^{2}} & -\frac{\alpha^{2} \beta}{\gamma^{2}} & -\frac{\alpha^{2}}{\gamma}
\end{array}\right)
$$


If we take the change of basis $B^{\prime}=\left\{\frac{1}{\alpha} e_{1}, \frac{\beta}{\alpha^{2}} e_{2}, \frac{\gamma}{\alpha^{2}} e_{3}\right\}$ then

$$
M_{B^{\prime}}=\left(\begin{array}{ccc}
1 & 1 & 1 \\
0 & 0 & 0 \\
-1 & -1 & -1
\end{array}\right) \text {. }
$$

Let $B^{\prime \prime}=\left\{-e_{3},-e_{1}-e_{2}, e_{2}\right\}$, then by this change of basis we have

$$
M_{B^{\prime \prime}}=\left(\begin{array}{ccc}
1 & 1 & 0 \\
-1 & -1 & 0 \\
0 & 0 & 0
\end{array}\right) .
$$

So, in this case also $E_{M_{B}}$ is isomorphic to $E_{1}$.

Case 1.1.3. Let $\gamma=0, \lambda \beta \mu \neq 0$. Then $\alpha^{2}+\beta^{2} \lambda=0$ and $\lambda=-\frac{\alpha^{2}}{\beta^{2}}$. In this case the structure matrix is:

$$
M_{B}=\left(\begin{array}{ccc}
\alpha & \beta & 0 \\
-\frac{\alpha^{3}}{\beta^{2}} & -\frac{\alpha^{2}}{\beta} & 0 \\
\mu \alpha & \mu \beta & 0
\end{array}\right) .
$$

Assume $\mu>0$. If we take the change of basis $B^{\prime}=\left\{\frac{1}{\alpha} e_{1}, \frac{\beta}{\alpha^{2}} e_{2}, \frac{1}{\alpha \sqrt{\mu}} e_{3}\right\}$ then

$$
M_{B^{\prime}}=\left(\begin{array}{ccc}
1 & 1 & 0 \\
-1 & -1 & 0 \\
1 & 1 & 0
\end{array}\right) \text {. }
$$

Thus in this case $E_{M_{B}}$ is isomorphic to $E_{2}$.

Assume $\mu<0$. If we take $B^{\prime}=\left\{\frac{1}{\alpha} e_{1}, \frac{\beta}{\alpha^{2}} e_{2}, \frac{1}{\alpha \sqrt{-\mu}} e_{3}\right\}$ then

$$
M_{B^{\prime}}=\left(\begin{array}{ccc}
1 & 1 & 0 \\
-1 & -1 & 0 \\
-1 & -1 & 0
\end{array}\right) \text {. }
$$

In this case $E_{M_{B}}$ is isomorphic to $E_{3}$.

Case 1.1.4. Let $\beta=0, \lambda \gamma \mu \neq 0$. Then $\alpha^{2}+\gamma^{2} \mu=0$ and $\mu=-\frac{\alpha^{2}}{\gamma^{2}}$. We have

$$
M_{B}=\left(\begin{array}{ccc}
\alpha & 0 & \gamma \\
\lambda \alpha & 0 & \lambda \gamma \\
-\frac{\alpha^{3}}{\gamma^{2}} & 0 & -\frac{\alpha^{2}}{\gamma}
\end{array}\right) .
$$

If we take the basis $B^{\prime}=\left\{e_{1}, e_{3}, e_{2}\right\}$ then

$$
M_{B^{\prime}}=\left(\begin{array}{ccc}
\alpha & \gamma & 0 \\
-\frac{\alpha^{3}}{\gamma^{2}} & -\frac{\alpha^{2}}{\gamma} & 0 \\
\lambda \alpha & \lambda \gamma & 0
\end{array}\right) .
$$

This case is similar to the case 1.1.3. $E_{M_{B}}$ is isomorphic to $E_{2}$ when $\lambda>0$, isomorphic to $E_{3}$ when $\lambda<0$. 
Case 1.1.5. Let $\gamma=\mu=0, \lambda \beta \neq 0$. Then $\alpha^{2}+\beta^{2} \lambda=0$ and $\lambda=-\frac{\alpha^{2}}{\beta^{2}}$. In this case the structure matrix is:

$$
M_{B}=\left(\begin{array}{ccc}
\alpha & \beta & 0 \\
-\frac{\alpha^{3}}{\beta^{2}} & -\frac{\alpha^{2}}{\beta} & 0 \\
0 & 0 & 0
\end{array}\right) .
$$

If we take the basis $B^{\prime}=\left\{\frac{1}{\alpha} e_{1}, \frac{\beta}{\alpha^{2}} e_{2}, e_{3}\right\}$ then

$$
M_{B^{\prime}}=\left(\begin{array}{ccc}
1 & 1 & 0 \\
-1 & -1 & 0 \\
0 & 0 & 0
\end{array}\right) \text {. }
$$

In this case $E_{M_{B}}$ is isomorphic to $E_{1}$.

Case 1.1.6. Let $\lambda=\beta=0, \gamma \mu \neq 0$. Taking the change of basis $B^{\prime}=\left\{e_{1}, e_{3}, e_{2}\right\}$ then we are in the case 1.1.5.

Case 1.1.7. Let $\lambda \beta \gamma \mu \neq 0$. If we take the basis $B^{\prime}=\left\{\frac{1}{\alpha} e_{1}, \frac{\beta}{\alpha^{2}} e_{2}, \frac{\gamma}{\alpha^{2}} e_{3}\right\}$ then

$$
M_{B^{\prime}}=\left(\begin{array}{ccc}
1 & 1 & 1 \\
\frac{\lambda \beta^{2}}{\alpha^{2}} & \frac{\lambda \beta^{2}}{\alpha^{2}} & \frac{\lambda \beta^{2}}{\alpha^{2}} \\
\frac{\mu \gamma^{2}}{\alpha^{2}} & \frac{\mu \gamma^{2}}{\alpha^{2}} & \frac{\mu \gamma^{2}}{\alpha^{2}}
\end{array}\right) .
$$

From the equality $\alpha^{2}+\beta^{2} \lambda+\gamma^{2} \mu=0$ we have $\frac{\lambda \beta^{2}}{\alpha^{2}}=-1-\frac{\mu \gamma^{2}}{\alpha^{2}}$. Hence

$$
M_{B^{\prime}}=\left(\begin{array}{ccc}
1 & 1 & 1 \\
-1-\frac{\mu \gamma^{2}}{\alpha^{2}} & -1-\frac{\mu \gamma^{2}}{\alpha^{2}} & -1-\frac{\mu \gamma^{2}}{\alpha^{2}} \\
\frac{\mu \gamma^{2}}{\alpha^{2}} & \frac{\mu \gamma^{2}}{\alpha^{2}} & \frac{\mu \gamma^{2}}{\alpha^{2}}
\end{array}\right) .
$$

Now, we take the change of basis $B^{\prime \prime}=\left\{e_{1}^{\prime}, e_{2}^{\prime}, e_{3}^{\prime}\right\}$ such that which the matrix is following

$$
P_{B^{\prime \prime} B^{\prime}}=\left(\begin{array}{ccc}
\frac{\alpha^{2}(K+1)}{2\left(\alpha^{2}+\mu \gamma^{2}\right)} & \frac{\alpha^{2}(K-1)}{2\left(\alpha^{2}+\mu \gamma^{2}\right)} & \frac{\alpha^{2}(K+1)}{2\left(\alpha^{2}+\mu \gamma^{2}\right)} \\
\frac{\alpha^{2}(K-1)}{2\left(\alpha^{2}+\mu \gamma^{2}\right)} & \frac{\alpha^{2}(K+1)}{2\left(\alpha^{2}+\mu \gamma^{2}\right)} & \frac{\alpha^{2}(K-1)}{2\left(\alpha^{2}+\mu \gamma^{2}\right)} \\
-\frac{\mu \gamma^{2}}{\alpha^{2}} & 0 & 1
\end{array}\right),
$$

where $K=\frac{\mu \gamma^{2}}{\alpha^{2}}\left(1+\frac{\mu \gamma^{2}}{\alpha^{2}}\right)^{2}$ and note that $\left|P_{B^{\prime \prime} B^{\prime}}\right|=\frac{\mu \gamma^{2}\left(\alpha^{2}+\mu \gamma^{2}\right)}{\alpha^{4}} \neq 0$. Then we obtain

$$
M_{B^{\prime \prime}}=\left(\begin{array}{ccc}
1 & 1 & 0 \\
-1 & -1 & 0 \\
1 & 1 & 0
\end{array}\right) \text {. }
$$

In this case $E_{M_{B}}$ is isomorphic to $E_{2}$.

Case 1.2. Assume that $\alpha^{2}+\beta^{2} \lambda+\gamma^{2} \mu \neq 0$. We consider the next cases.

Case 1.2.1. Suppose $\lambda=0, \mu=0$. In this case the structure matrix is:

$$
M_{B}=\left(\begin{array}{ccc}
\alpha & \beta & \gamma \\
0 & 0 & 0 \\
0 & 0 & 0
\end{array}\right) \text {. }
$$


Consider the change of basis $B^{\prime}=\left\{\frac{1}{\alpha} e_{1}+\frac{\beta}{\alpha^{2}} e_{2}+\frac{\gamma}{\alpha^{2}} e_{3} ; e_{2}-e_{3} ; 4 e_{2}+e_{3}\right\}$. Then

$$
M_{B^{\prime}}=\left(\begin{array}{lll}
1 & 0 & 0 \\
0 & 0 & 0 \\
0 & 0 & 0
\end{array}\right) \text {. }
$$

In this case $E_{M_{B}}$ is isomorphic to $E_{4}$.

Case 1.2.2. Suppose $\lambda=0, \mu \neq 0$. Then $\alpha^{2}+\gamma^{2} \mu \neq 0$.

For $B^{\prime}=\left\{\frac{1}{\alpha} e_{1}+\frac{\beta}{\alpha^{2}} e_{2}+\frac{\gamma}{\alpha^{2}} e_{3} ; e_{2} ;-\frac{\gamma \mu}{\alpha} e_{1}+e_{2}+e_{3}\right\}$ the structure matrix is

$$
M_{B^{\prime}}=\left(\begin{array}{ccc}
1+\frac{\mu \gamma^{2}}{\alpha^{2}} & 0 & 0 \\
0 & 0 & 0 \\
\mu\left(\alpha^{2}+\mu \gamma^{2}\right) & 0 & 0
\end{array}\right)
$$

Case 1.2.2.1. Assume that $\mu>0$. Consider $B^{\prime \prime}=\left\{\frac{\alpha^{2}}{\alpha^{2}+\mu \gamma^{2}} e_{1} ; e_{2} ; \frac{\alpha}{\sqrt{\mu}\left(\alpha^{2}+\mu \gamma^{2}\right)} e_{3}\right\}$. Then $M_{B^{\prime \prime}}=\left(\begin{array}{lll}1 & 0 & 0 \\ 0 & 0 & 0 \\ 1 & 0 & 0\end{array}\right)$. In this case $E_{M_{B}}$ is isomorphic to $E_{5}$.

Case 1.2.2.2. Assume that $\mu<0$. Consider $B^{\prime \prime}=\left\{\frac{\alpha^{2}}{\alpha^{2}+\mu \gamma^{2}} e_{1} ; e_{2} ; \frac{\alpha}{\sqrt{-\mu}\left(\alpha^{2}+\mu \gamma^{2}\right)} e_{3}\right\}$. Then $M_{B^{\prime \prime}}=\left(\begin{array}{ccc}1 & 0 & 0 \\ 0 & 0 & 0 \\ -1 & 0 & 0\end{array}\right)$. In this case $E_{M_{B}}$ is isomorphic to $E_{6}$.

Case 1.2.3. Assume that $\lambda \neq 0$ and $\mu=0$. If we take the basis $B^{\prime}=\left\{e_{1}, e_{3}, e_{2}\right\}$ then we are in the same conditions as in Case 1.2.2. Namely, $E_{M_{B}}$ is isomorphic to $E_{5}$ and $E_{6}$ respectively $\lambda>0$ and $\lambda<0$.

Case 1.2.4. Assume that $\lambda \neq 0, \mu \neq 0$ and $\alpha^{2}+\lambda \beta^{2} \neq 0$. If $B^{\prime}$ is the change of basis such that

$$
P_{B^{\prime} B}=\left(\begin{array}{ccc}
\frac{1}{\alpha} & \frac{\beta}{\alpha^{2}} & \frac{\gamma}{\alpha^{2}} \\
-\frac{\lambda \beta}{\alpha} & 1 & 0 \\
\frac{-\mu \gamma}{\alpha^{2}+\lambda \beta^{2}} & \frac{-\beta \mu \gamma}{\alpha\left(\alpha^{2}+\lambda \beta^{2}\right)} & \frac{1}{\alpha}
\end{array}\right)
$$

then we obtain

$$
M_{B^{\prime}}=\left(\begin{array}{ccc}
\frac{1}{\alpha^{2}}\left(\alpha^{2}+\lambda \beta^{2}+\mu \gamma^{2}\right) & 0 & 0 \\
\lambda\left(\alpha^{2}+\lambda \beta^{2}\right) & 0 & 0 \\
\frac{\mu\left(\alpha^{2}+\lambda \beta^{2}+\mu \gamma^{2}\right)}{\alpha^{2}+\lambda \beta^{2}} & 0 & 0
\end{array}\right)
$$

Note that $\left|P_{B^{\prime} B}\right|=\frac{1}{\alpha^{4}}\left(\alpha^{2}+\lambda \beta^{2}+\mu \gamma^{2}\right) \neq 0$.

Case 1.2.4.1. If $\lambda>0$ and $\mu>0$ then consider the change of basis $B^{\prime \prime}=\left\{e_{1}^{\prime \prime}, e_{2}^{\prime \prime}, e_{3}^{\prime \prime}\right\}$

$$
P_{B^{\prime \prime} B^{\prime}}=\left(\begin{array}{ccc}
\frac{\alpha^{2}}{\alpha^{2}+\lambda \beta^{2}+\mu \gamma^{2}} & 0 & 0 \\
0 & \frac{\alpha}{\sqrt{\lambda\left(\alpha^{2}+\lambda \beta^{2}\right)\left(\alpha^{2}+\lambda \beta^{2}+\mu \gamma^{2}\right)}} & 0 \\
0 & 0 & \frac{\alpha \sqrt{\alpha^{2}+\lambda \beta^{2}}}{\sqrt{\mu}\left(\alpha^{2}+\lambda \beta^{2}+\mu \gamma^{2}\right)}
\end{array}\right)
$$


and the structure matrix is

$$
M_{B^{\prime \prime}}=\left(\begin{array}{ccc}
1 & 0 & 0 \\
1 & 0 & 0 \\
1 & 0 & 0
\end{array}\right)
$$

In this case $E_{M_{B}}$ is isomorphic to $E_{7}$.

Case 1.2.4.2. If $\lambda>0$ and $\mu<0$. Assume that $\alpha^{2}+\lambda \beta^{2}+\mu \gamma^{2}>0$. Consider the change of basis $B^{\prime \prime}=\left\{e_{1}^{\prime \prime}, e_{2}^{\prime \prime}, e_{3}^{\prime \prime}\right\}$

$$
P_{B^{\prime \prime} B^{\prime}}=\left(\begin{array}{ccc}
\frac{\alpha^{2}}{\alpha^{2}+\lambda \beta^{2}+\mu \gamma^{2}} & 0 & 0 \\
0 & \frac{\alpha}{\sqrt{\lambda\left(\alpha^{2}+\lambda \beta^{2}\right)\left(\alpha^{2}+\lambda \beta^{2}+\mu \gamma^{2}\right)}} & 0 \\
0 & 0 & \frac{\alpha \sqrt{\alpha^{2}+\lambda \beta^{2}}}{\sqrt{-\mu}\left(\alpha^{2}+\lambda \beta^{2}+\mu \gamma^{2}\right)}
\end{array}\right)
$$

and the structure matrix is

$$
M_{B^{\prime \prime}}=\left(\begin{array}{ccc}
1 & 0 & 0 \\
1 & 0 & 0 \\
-1 & 0 & 0
\end{array}\right)
$$

In this case $E_{M_{B}}$ is isomorphic to $E_{8}$.

Case 1.2.4.3. If $\lambda>0$ and $\mu<0$. Assume that $\alpha^{2}+\lambda \beta^{2}+\mu \gamma^{2}<0$. Consider the change of basis $B^{\prime \prime}=\left\{e_{1}^{\prime \prime}, e_{2}^{\prime \prime}, e_{3}^{\prime \prime}\right\}$

$$
P_{B^{\prime \prime} B^{\prime}}=\left(\begin{array}{ccc}
\frac{\alpha^{2}}{\alpha^{2}+\lambda \beta^{2}+\mu \gamma^{2}} & 0 & 0 \\
0 & \frac{\alpha}{\sqrt{-\lambda\left(\alpha^{2}+\lambda \beta^{2}\right)\left(\alpha^{2}+\lambda \beta^{2}+\mu \gamma^{2}\right)}} & 0 \\
0 & 0 & \frac{\alpha \sqrt{\alpha^{2}+\lambda \beta^{2}}}{\sqrt{-\mu}\left(\alpha^{2}+\lambda \beta^{2}+\mu \gamma^{2}\right)}
\end{array}\right)
$$

and the structure matrix is

$$
M_{B^{\prime \prime}}=\left(\begin{array}{ccc}
1 & 0 & 0 \\
-1 & 0 & 0 \\
-1 & 0 & 0
\end{array}\right)
$$

In this case $E_{M_{B}}$ is isomorphic to $E_{9}$.

Case 1.2.4.4. If $\lambda<0$ and $\mu>0$. This case is similar to the Case 1.2.4.2 and Case 1.2.4.3. There are only differences in conditions.

The structure matrix is $\left(\begin{array}{ccc}1 & 0 & 0 \\ 1 & 0 & 0 \\ -1 & 0 & 0\end{array}\right)$ when $\alpha^{2}+\lambda \beta^{2}<0$ and $\alpha^{2}+\lambda \beta^{2}+\mu \gamma^{2}>0$.

The structure matrix is $\left(\begin{array}{ccc}1 & 0 & 0 \\ -1 & 0 & 0 \\ -1 & 0 & 0\end{array}\right)$ when $\alpha^{2}+\lambda \beta^{2}<0$ and $\alpha^{2}+\lambda \beta^{2}+\mu \gamma^{2}<0$. 
The structure matrix is $\left(\begin{array}{ccc}1 & 0 & 0 \\ -1 & 0 & 0 \\ 1 & 0 & 0\end{array}\right)$ when $\alpha^{2}+\lambda \beta^{2}>0$ and if we take the change of basis $\left\{e_{1}, e_{3}, e_{2}\right\}$ then we have $\left(\begin{array}{ccc}1 & 0 & 0 \\ 1 & 0 & 0 \\ -1 & 0 & 0\end{array}\right)$.

Case 1.2.4.5. If $\lambda<0$ and $\mu<0$. In this case the same as above, There are differences in conditions.

The structure matrix is $\left(\begin{array}{ccc}1 & 0 & 0 \\ 1 & 0 & 0 \\ -1 & 0 & 0\end{array}\right)$ when $\alpha^{2}+\lambda \beta^{2}>0$ and $\alpha^{2}+\lambda \beta^{2}+\mu \gamma^{2}<0$.

The structure matrix is $\left(\begin{array}{ccc}1 & 0 & 0 \\ -1 & 0 & 0 \\ -1 & 0 & 0\end{array}\right)$ when $\alpha^{2}+\lambda \beta^{2}>0$ and $\alpha^{2}+\lambda \beta^{2}+\mu \gamma^{2}>0$.

The structure matrix is $\left(\begin{array}{ccc}1 & 0 & 0 \\ -1 & 0 & 0 \\ 1 & 0 & 0\end{array}\right)$ when $\alpha^{2}+\lambda \beta^{2}<0$ and if we take the change of basis $\left\{e_{1}, e_{3}, e_{2}\right\}$ then we have $\left(\begin{array}{ccc}1 & 0 & 0 \\ 1 & 0 & 0 \\ -1 & 0 & 0\end{array}\right)$.

Case 1.2.5. Suppose that $\lambda \neq 0, \mu \neq 0$ and $\alpha^{2}+\lambda \beta^{2}=0$. Then $\lambda \beta \mu \gamma \neq 0$ and so $\lambda=-\frac{\alpha^{2}}{\beta^{2}}$. If we take the change of basis $B^{\prime}=\left\{e_{1}^{\prime}, e_{2}^{\prime}, e_{3}^{\prime}\right\}$ such that

$$
P_{B^{\prime} B}=\left(\begin{array}{ccc}
\frac{1}{\alpha} & \frac{\beta}{\alpha^{2}} & \frac{\gamma}{\alpha^{2}} \\
-\frac{\mu}{2 \alpha} & \frac{\beta \mu}{2 \alpha^{2}} & \frac{1}{\gamma} \\
\frac{2 \alpha^{2}-\mu \gamma^{2}}{2 \alpha^{3}} & \frac{\beta\left(2 \alpha^{2}+\mu \gamma^{2}\right)}{2 \alpha^{4}} & \frac{\gamma}{\alpha^{2}}
\end{array}\right)
$$

then we obtain

$$
\begin{gathered}
e_{1}^{\prime} e_{1}^{\prime}=\frac{1}{\alpha^{2}} e_{1}^{2}+\frac{\beta^{2}}{\alpha^{4}} e_{2}^{2}+\frac{\gamma^{2}}{\alpha^{4}} e_{3}^{2}=\left(\frac{1}{\alpha^{2}}+\lambda \frac{\beta^{2}}{\alpha^{4}}+\mu \frac{\gamma^{2}}{\alpha^{4}}\right)\left(\alpha e_{1}+\beta e_{2}+\gamma e_{3}\right)=\frac{\mu \gamma^{2}}{\alpha^{2}} e_{1}^{\prime}, \\
e_{2}^{\prime} e_{2}^{\prime}=\frac{\mu^{2}}{4 \alpha^{2}} e_{1}^{2}+\frac{\beta^{2} \mu^{2}}{4 \alpha^{4}} e_{2}^{2}+\frac{1}{\gamma^{2}} e_{3}^{2}=\left(\frac{\mu^{2}}{4 \alpha^{2}}+\lambda \frac{\beta^{2} \mu^{2}}{4 \alpha^{4}}+\frac{\mu}{\gamma^{2}}\right)\left(\alpha e_{1}+\beta e_{2}+\gamma e_{3}\right)=\frac{\mu \alpha^{2}}{\gamma^{2}} e_{1}^{\prime}, \\
e_{3}^{\prime} e_{3}^{\prime}=\frac{\left(2 \alpha^{2}-\mu \gamma^{2}\right)^{2}}{4 \alpha^{6}} e_{1}^{2}+\frac{\beta^{2}\left(2 \alpha^{2}+\mu \gamma^{2}\right)^{2}}{4 \alpha^{8}} e_{2}^{2}+\frac{\gamma^{2}}{\alpha^{4}} e_{3}^{2}=\left(\frac{\left(2 \alpha^{2}-\mu \gamma^{2}\right)^{2}}{4 \alpha^{6}}+\lambda \frac{\beta^{2}\left(2 \alpha^{2}+\mu \gamma^{2}\right)^{2}}{4 \alpha^{8}}+\right. \\
\left.+\mu \frac{\gamma^{2}}{\alpha^{4}}\right)\left(\alpha e_{1}+\beta e_{2}+\gamma e_{3}\right)=-\frac{\mu \gamma^{2}}{\alpha^{4}}\left(\alpha e_{1}+\beta e_{2}+\gamma e_{3}\right)=-\frac{\mu \gamma^{2}}{\alpha^{2}} e_{1}^{\prime} .
\end{gathered}
$$

Moreover

$$
\begin{gathered}
e_{1}^{\prime} e_{2}^{\prime}=-\frac{\mu}{2 \alpha^{2}} e_{1}^{2}+\frac{\beta^{2} \mu}{2 \alpha^{4}} e_{2}^{2}+\frac{1}{\alpha^{2}} e_{3}^{2}=e_{1}^{2}\left(-\frac{\mu}{2 \alpha^{2}}+\lambda \frac{\beta^{2} \mu}{2 \alpha^{4}}+\frac{\mu}{\alpha^{2}}\right)=e_{1}^{2}\left(\lambda \frac{\beta^{2} \mu}{2 \alpha^{4}}+\frac{\mu}{2 \alpha^{2}}\right)=0 \\
e_{1}^{\prime} e_{3}^{\prime}=\frac{2 \alpha^{2}-\mu \gamma^{2}}{2 \alpha^{4}} e_{1}^{2}+\frac{\beta^{2}\left(2 \alpha^{2}+\mu \gamma^{2}\right)}{2 \alpha^{6}} e_{2}^{2}+\frac{\gamma^{2}}{\alpha^{4}} e_{3}^{2}=\frac{2 \alpha^{2}\left(\alpha^{2}+\lambda \beta^{2}\right)+\mu \gamma^{2}\left(\alpha^{2}+\lambda \beta^{2}\right)}{2 \alpha^{6}} e_{1}^{2}=0 \\
e_{2}^{\prime} e_{3}^{\prime}=\frac{-\mu\left(2 \alpha^{2}-\mu \gamma^{2}\right)}{4 \alpha^{4}} e_{1}^{2}+\frac{\mu \beta^{2}\left(2 \alpha^{2}+\mu \gamma^{2}\right)}{4 \alpha^{6}} e_{2}^{2}+\frac{1}{\alpha^{2}} e_{3}^{2}=e_{1}^{2}\left(\frac{-2 \mu \alpha^{2}+\mu^{2} \gamma^{2}}{4 \alpha^{4}}+\frac{2 \alpha^{2} \mu \lambda \beta^{2}+\mu^{2} \gamma^{2} \lambda \beta^{2}}{4 \alpha^{6}}+\right. \\
\left.+\frac{\mu}{\alpha^{2}}\right)=e_{1}^{2}\left(\frac{-2 \mu \alpha^{4}+2 \mu \alpha^{2} \lambda \beta^{2}}{4 \alpha^{6}}+\frac{\mu}{\alpha^{2}}\right)=\frac{-2 \mu \alpha^{4}+2 \mu \alpha^{2} \lambda \beta^{2}+4 \mu \alpha^{4}}{4 \alpha^{6}} e_{1}^{2}=\frac{2 \mu \alpha^{2}\left(\alpha^{2}+\lambda \beta^{2}\right)}{4 \alpha^{6}}=0,
\end{gathered}
$$


note that $\left|P_{B^{\prime} B}\right|=-\frac{\beta \mu \gamma}{\alpha^{5}} \neq 0$. So, we have

$$
M_{B^{\prime}}=\left(\begin{array}{ccc}
\frac{\mu \gamma^{2}}{\alpha^{2}} & 0 & 0 \\
\frac{\mu}{\gamma^{2}} \alpha^{2} & 0 & 0 \\
-\frac{\mu \gamma^{2}}{\alpha^{2}} & 0 & 0
\end{array}\right) .
$$

Considering the change of basis $B^{\prime \prime}=\left\{\frac{\alpha^{2}}{\mu \gamma^{2}} e_{1}, \frac{1}{\mu} e_{2}, \frac{-\alpha^{2}}{\mu \gamma^{2}} e_{3}\right\}$ and we obtain

$$
M_{B^{\prime \prime}}=\left(\begin{array}{ccc}
1 & 0 & 0 \\
1 & 0 & 0 \\
-1 & 0 & 0
\end{array}\right) \text {. }
$$

In this case $E_{M_{B}}$ is isomorphic to $E_{8}$.

Case 2. Suppose that $\alpha=0$. In this case the structure matrix of the evolution algebra is

$$
M_{B}=\left(\begin{array}{ccc}
0 & \beta & \gamma \\
0 & \lambda \beta & \lambda \gamma \\
0 & \mu \beta & \mu \gamma
\end{array}\right) .
$$

Necessarily that $\beta^{2}+\gamma^{2} \neq 0$. Without loss of generality we assume $\beta \neq 0$.

Case 2.1. Assume $\lambda \neq 0$. Consider the change of basis $B^{\prime}=\left\{e_{2}, e_{3}, e_{1}\right\}$. Then

$$
M_{B^{\prime}}=\left(\begin{array}{ccc}
\lambda \beta & \lambda \gamma & 0 \\
\mu \beta & \mu \gamma & 0 \\
\beta & \gamma & 0
\end{array}\right) .
$$

Case 2.1.1. Suppose $\mu=0, \gamma \neq 0$ and $\lambda>0$. Consider the change of basis $B^{\prime \prime}=\left\{e_{1}^{\prime \prime}, e_{2}^{\prime \prime}, e_{3}^{\prime \prime}\right\}$ such that

$$
P_{B^{\prime \prime} B^{\prime}}=\left(\begin{array}{ccc}
\frac{1}{\lambda \beta} & \frac{\gamma}{\lambda \beta^{2}} & 0 \\
0 & 1 & 0 \\
0 & 0 & \frac{\sqrt{\lambda}}{\lambda \beta}
\end{array}\right) .
$$

We have

$$
M_{B^{\prime \prime}}=\left(\begin{array}{ccc}
1 & 0 & 0 \\
0 & 0 & 0 \\
1 & 0 & 0
\end{array}\right) .
$$

In this case $E_{M_{B}}$ is isomorphic to $E_{5}$.

If $\lambda<0$ then we take as $B^{\prime \prime}=\left\{e_{1}^{\prime \prime}, e_{2}^{\prime \prime}, e_{3}^{\prime \prime}\right\}$ the following

$$
P_{B^{\prime \prime} B^{\prime}}=\left(\begin{array}{ccc}
\frac{1}{\lambda \beta} & \frac{\gamma}{\lambda \beta^{2}} & 0 \\
0 & 1 & 0 \\
0 & 0 & \frac{\sqrt{-\lambda}}{\lambda \beta}
\end{array}\right) \text {. }
$$

We have

$$
M_{B^{\prime \prime}}=\left(\begin{array}{ccc}
1 & 0 & 0 \\
0 & 0 & 0 \\
-1 & 0 & 0
\end{array}\right) .
$$


In this case $E_{M_{B}}$ is isomorphic to $E_{6}$.

Case 2.1.2. The cases $\mu=0, \gamma=0, \lambda>0$ and $\mu=0, \gamma=0, \lambda<0$ are similar to the previous case. In this case $E_{M_{B}}$ is isomorphic to $E_{5}$ and $E_{6}$ respectively.

Case 2.1.3. Assume $\mu \neq 0, \gamma=0$. In this case if $\lambda>0, \mu>0$ then for $B^{\prime \prime}=$ $\left\{\frac{1}{\lambda \beta} e_{1} ; \frac{1}{\sqrt{\mu \lambda} \beta} e_{2} ; \frac{1}{\sqrt{\lambda} \beta} e_{3}\right\}$ we have

$$
M_{B^{\prime \prime}}=\left(\begin{array}{lll}
1 & 0 & 0 \\
1 & 0 & 0 \\
1 & 0 & 0
\end{array}\right) .
$$

In this case $E_{M_{B}}$ is isomorphic to $E_{7}$.

Similarly, if $\lambda<0, \mu<0$ and $\lambda>0, \mu<0$ then the structure matrix will be

$$
M_{B^{\prime \prime}}=\left(\begin{array}{ccc}
1 & 0 & 0 \\
1 & 0 & 0 \\
-1 & 0 & 0
\end{array}\right) .
$$

In this case $E_{M_{B}}$ is isomorphic to $E_{8}$.

If $\lambda<0, \mu>0$ then the structure matrix will be

$$
M_{B^{\prime \prime}}=\left(\begin{array}{ccc}
1 & 0 & 0 \\
-1 & 0 & 0 \\
-1 & 0 & 0
\end{array}\right) .
$$

In this case $E_{M_{B}}$ is isomorphic to $E_{9}$.

Case 2.1.4. Assume $\mu \neq 0, \gamma \neq 0$. Now, consider the change of basis $B^{\prime \prime}=$ $\left\{\frac{1}{\lambda \beta} e_{1} ; e_{2} ; e_{3}\right\}$. Then

$$
M_{B^{\prime \prime}}=\left(\begin{array}{ccc}
1 & \frac{\gamma}{\lambda \beta^{2}} & 0 \\
\mu \lambda \beta^{2} & \mu \gamma & 0 \\
\lambda \beta^{2} & \gamma & 0
\end{array}\right) .
$$

Case 2.1.4.1. Suppose that $\lambda \beta^{2}+\mu \gamma^{2}=0$. And so $\mu=-\frac{\lambda \beta^{2}}{\gamma^{2}}$. It follows that

$$
M_{B^{\prime \prime}}=\left(\begin{array}{ccc}
1 & \frac{\gamma}{\lambda \beta^{2}} & 0 \\
-\frac{\lambda^{2} \beta^{4}}{\gamma^{2}} & -\frac{\lambda \beta^{2}}{\gamma} & 0 \\
\lambda \beta^{2} & \gamma & 0
\end{array}\right) .
$$

Let's $\lambda>0$, if we take $B^{\prime \prime \prime}=\left\{e_{1} ; \frac{\gamma}{\lambda \beta^{2}} e_{2}, \frac{1}{\sqrt{\lambda} \beta}\right\}$ then $M_{B^{\prime \prime \prime}}=\left(\begin{array}{ccc}1 & 1 & 0 \\ -1 & -1 & 0 \\ 1 & 1 & 0\end{array}\right)$.

In this case $E_{M_{B}}$ is isomorphic to $E_{2}$.

Let's $\lambda<0$, in this case we may take $B^{\prime \prime \prime}=\left\{e_{1} ; \frac{\gamma}{\lambda \beta^{2}} e_{2}, \frac{1}{\sqrt{\lambda} \beta}\right\}$, then we have $M_{B^{\prime \prime \prime}}=\left(\begin{array}{ccc}1 & 1 & 0 \\ -1 & -1 & 0 \\ -1 & -1 & 0\end{array}\right)$. In this case $E_{M_{B}}$ is isomorphic to $E_{3}$. 
Case 2.1.4.2. Suppose that $\lambda \beta^{2}+\mu \gamma^{2} \neq 0$ and $\left\{\begin{array}{l}\lambda \mu>0 \\ \lambda \beta^{2}+\mu \gamma^{2}>0\end{array}\right.$. If we take $B^{\prime \prime \prime}$ is the change of basis such that

$$
P_{B^{\prime \prime \prime} B^{\prime \prime}}=\left(\begin{array}{ccc}
1 & \frac{\gamma}{\lambda \beta^{2}} & 0 \\
-\mu \gamma & 1 & 0 \\
0 & 0 & 1
\end{array}\right)
$$

then we obtain that

$$
M_{B^{\prime \prime \prime}}=\left(\begin{array}{ccc}
\frac{\lambda \beta^{2}+\mu \gamma^{2}}{\lambda \beta^{2}} & 0 & 0 \\
\mu\left(\lambda \beta^{2}+\mu \gamma^{2}\right) & 0 & 0 \\
\lambda \beta^{2} & 0 & 0
\end{array}\right)
$$

Now, consider the change of basis $B^{*}=\left\{e_{1}^{*}, e_{2}^{*}, e_{3}^{*}\right\}$ such that

$$
P_{B^{*} B^{\prime \prime \prime}}=\left(\begin{array}{ccc}
\frac{\lambda \beta^{2}}{\lambda \beta^{2}+\mu \gamma^{2}} & 0 & 0 \\
0 & \sqrt{\frac{\lambda}{\mu}} \frac{\beta}{\lambda \beta^{2}+\mu \gamma^{2}} & 0 \\
0 & 0 & \frac{1}{\sqrt{\lambda \beta^{2}+\mu \gamma^{2}}}
\end{array}\right)
$$

and the structure matrix is $M_{B^{*}}=\left(\begin{array}{ccc}1 & 0 & 0 \\ 1 & 0 & 0 \\ 1 & 0 & 0\end{array}\right)$. In this case $E_{M_{B}}$ is isomorphic to $E_{7}$. Similarly, it is not difficult to show that if $\left\{\begin{array}{l}\lambda \mu<0 \\ \lambda \beta^{2}+\mu \gamma^{2}>0\end{array}\right.$ and $\left\{\begin{array}{l}\lambda \mu>0 \\ \lambda \beta^{2}+\mu \gamma^{2}<0\end{array}\right.$ then the structure matrix is $M_{B^{*}}=\left(\begin{array}{ccc}1 & 0 & 0 \\ 1 & 0 & 0 \\ -1 & 0 & 0\end{array}\right)$. In this case $E_{M_{B}}$ is isomorphic to $E_{8}$.

If $\left\{\begin{array}{l}\lambda \mu<0 \\ \lambda \beta^{2}+\mu \gamma^{2}<0\end{array}\right.$ then the structure matrix is $M_{B^{*}}=\left(\begin{array}{ccc}1 & 0 & 0 \\ -1 & 0 & 0 \\ -1 & 0 & 0\end{array}\right)$. In this case $E_{M_{B}}$ is isomorphic to $E_{9}$.

Case 2.2. Now, we consider the case $\lambda=0$.

Case 2.2.1. Assume that $\mu \gamma \neq 0$. Consider the change of basis $B^{\prime}\left\{e_{3}, e_{2}, e_{1}\right\}$ and we obtain

$$
M_{B^{\prime}}=\left(\begin{array}{ccc}
\mu \gamma & \mu \beta & 0 \\
0 & 0 & 0 \\
\gamma & \beta & 0
\end{array}\right) .
$$

If we take $B^{\prime \prime}=\left\{\mu \gamma e_{1}+\mu \beta e_{2} ; e_{2}, \mu e_{3}\right\}$ then

$$
M_{B^{*}}=\left(\begin{array}{ccc}
\mu^{2} \gamma^{2} & 0 & 0 \\
0 & 0 & 0 \\
\mu & 0 & 0
\end{array}\right) .
$$


Let $\mu>0$, consider the change of basis $B^{\prime \prime \prime}=\left\{\frac{1}{\mu^{2} \gamma^{2}} e_{1} ; e_{2} ; \frac{1}{\sqrt{\mu} \mu \gamma} e_{3}\right\}$. Then $M_{B^{\prime \prime \prime}}=\left(\begin{array}{lll}1 & 0 & 0 \\ 0 & 0 & 0 \\ 1 & 0 & 0\end{array}\right)$. In this case $E_{M_{B}}$ is isomorphic to $E_{5}$.

Similarly, if $\mu<0$ then we have $M_{B^{\prime \prime \prime}}=\left(\begin{array}{ccc}1 & 0 & 0 \\ 0 & 0 & 0 \\ -1 & 0 & 0\end{array}\right)$. In this case $E_{M_{B}}$ is isomorphic to $E_{6}$.

Case 2.2.2. Suppose that $\mu \gamma=0$.

Case 2.2.2.1. Assume that $\mu=0$ and $\gamma$ is an arbitrary real number. Then the structure matrix is $M_{B}=\left(\begin{array}{ccc}0 & \beta & \gamma \\ 0 & 0 & 0 \\ 0 & 0 & 0\end{array}\right)$. Let's take the change of basis $B^{\prime}=\left\{\beta e_{2}+\gamma e_{3} ; \frac{1}{\beta} e_{3} ; e_{1}\right\}$ then $M_{B^{\prime}}=\left(\begin{array}{ccc}0 & 0 & 0 \\ 0 & 0 & 0 \\ 1 & 0 & 0\end{array}\right)$. In this case $E_{M_{B}}$ is isomorphic to $E_{10}$.

Case 2.2.2.2. Assume that $\mu>0$. It follows that $\gamma=0$. For $B^{\prime}=\left\{\beta e_{2} ; e_{1} ; \frac{1}{\sqrt{\mu}} e_{3}\right\}$ we have $M_{B^{\prime}}=\left(\begin{array}{lll}0 & 0 & 0 \\ 1 & 0 & 0 \\ 1 & 0 & 0\end{array}\right)$.

It means that in this case $E_{M_{B}}$ is isomorphic to $E_{11}$.

Case 2.2.2.3. Assume that $\mu<0$. It follows that $\gamma=0$. For $B^{\prime}=\left\{\beta e_{2} ; e_{1} ; \frac{1}{\sqrt{-\mu}} e_{3}\right\}$ we have $M_{B^{\prime}}=\left(\begin{array}{ccc}0 & 0 & 0 \\ 1 & 0 & 0 \\ -1 & 0 & 0\end{array}\right)$.

It means that in this case $E_{M_{B}}$ is isomorphic to $E_{12}$.

Using Lemma 3 we get the following theorem.

Theorem 3. For given values $(s, t) \in\left\{(s, t): \eta(s)+\varphi_{1}(s) \vartheta(s)+\varphi_{2}(s) \kappa(s) \neq 0, \eta(t) \neq 0\right.$, $\left.\eta^{2}(t)+\varphi_{1}(s) \vartheta^{2}(t)+\varphi_{2}(s) \kappa^{2}(t)=0\right\}$ of time, $E_{3}^{[s, t]}$ is isomorphic to

(a) $E_{1}$ if one of the following conditions is hold:

1) $\varphi_{2}(s)=0, \varphi_{1}(s) \vartheta(t) \kappa(t) \neq 0$;

2) $\varphi_{1}(s)=0, \varphi_{2}(s) \vartheta(t) \kappa(t) \neq 0$;

3) $\kappa(t)=\varphi_{2}(s)=0, \varphi_{1}(s) \vartheta(t) \neq 0$;

4) $\varphi_{1}(s)=\vartheta(t)=0, \kappa(t) \varphi_{2}(s) \neq 0$;

(b) $E_{2}$ if one of the following conditions is hold:

1) $\kappa(t)=0, \varphi_{1}(s) \varphi_{2}(s) \vartheta(t) \neq 0, \varphi_{2}(s)>0$;

2) $\vartheta(t)=0, \varphi_{1}(s) \varphi_{2}(s) \kappa(t) \neq 0, \varphi_{1}(s)>0$;

3) $\varphi_{1}(s) \varphi_{2}(s) \vartheta(t) \kappa(t) \neq 0$; 
(c) $E_{3}$ if one of the following conditions is hold:

1) $\kappa(t)=0, \varphi_{1}(s) \varphi_{2}(s) \vartheta(t) \neq 0, \varphi_{2}(s)<0$;

2) $\vartheta(t)=0, \varphi_{1}(s) \varphi_{2}(s) \kappa(t) \neq 0, \varphi_{1}(s)<0$;

For given values $(s, t) \in\left\{(s, t): \eta(s)+\varphi_{1}(s) \vartheta(s)+\varphi_{2}(s) \kappa(s) \neq 0, \eta(t) \neq 0, \eta^{2}(t)+\right.$ $\left.\varphi_{1}(s) \vartheta^{2}(t)+\varphi_{2}(s) \kappa^{2}(t) \neq 0\right\}$ of time, $E_{3}^{[s, t]}$ is isomorphic to

(d) $E_{4}$ if the following condition is hold:

$\varphi_{1}(s)=0, \varphi_{2}(s)=0$;

(e) $E_{5}$ if one of the following conditions is hold:

1) $\varphi_{1}(s)=0, \varphi_{2}(s)>0$;

2) $\varphi_{2}(s)=0, \varphi_{1}(s)>0$;

(f) $E_{6}$ if one of the following conditions is hold:

1) $\varphi_{1}(s)=0, \varphi_{2}(s)<0$;

2) $\varphi_{2}(s)=0, \varphi_{1}(s)<0$;

(g) $E_{7}$ if the following condition is hold: $\varphi_{1}(s)>0, \varphi_{2}(s)>0$;

(h) $E_{8}$ if one of the following conditions is hold:

1) $\varphi_{1}(s)>0, \varphi_{2}(s)<0, \eta^{2}(t)+\varphi_{1}(s) \vartheta^{2}(t)+\varphi_{2}(s) \kappa^{2}(t)>0$;

2) $\varphi_{1}(s)<0, \varphi_{2}(s)>0, \eta^{2}(t)+\varphi_{1}(s) \vartheta^{2}(t)<0, \eta^{2}(t)+\varphi_{1}(s) \vartheta^{2}(t)+\varphi_{2}(s) \kappa^{2}(t)>0$;

3) $\varphi_{1}(s)<0, \varphi_{2}(s)>0, \eta^{2}(t)+\varphi_{1}(s) \vartheta^{2}(t)>0$;

4) $\varphi_{1}(s)<0, \varphi_{2}(s)<0, \eta^{2}(t)+\varphi_{1}(s) \vartheta^{2}(t)>0, \eta^{2}(t)+\varphi_{1}(s) \vartheta^{2}(t)+\varphi_{2}(s) \kappa^{2}(t)<0$;

5) $\varphi_{1}(s)<0, \varphi_{2}(s)<0, \eta^{2}(t)+\varphi_{1}(s) \vartheta^{2}(t)<0$;

6) $\varphi_{1}(s) \neq 0, \varphi_{2}(s) \neq 0, \eta^{2}(t)+\varphi_{1}(s) \vartheta^{2}(t)=0$;

(i) $E_{9}$ if one of the following conditions is hold:

1) $\varphi_{1}(s)>0, \varphi_{2}(s)<0, \eta^{2}(t)+\varphi_{1}(s) \vartheta^{2}(t)+\varphi_{2}(s) \kappa^{2}(t)<0$;

2) $\varphi_{1}(s)<0, \varphi_{2}(s)>0, \eta^{2}(t)+\varphi_{1}(s) \vartheta^{2}(t)<0, \eta^{2}(t)+\varphi_{1}(s) \vartheta^{2}(t)+\varphi_{2}(s) \kappa^{2}(t)<0$;

3) $\varphi_{1}(s)<0, \varphi_{2}(s)<0, \eta^{2}(t)+\varphi_{1}(s) \vartheta^{2}(t)>0, \eta^{2}(t)+\varphi_{1}(s) \vartheta^{2}(t)+\varphi_{2}(s) \kappa^{2}(t)>0$.

For given values $(s, t) \in\left\{(s, t): \eta(s)+\varphi_{1}(s) \vartheta(s)+\varphi_{2}(s) \kappa(s) \neq 0, \eta(t)=0, \vartheta(t) \neq 0\right\}$ of time, $E_{3}^{[s, t]}$ is isomorphic to

(j) $E_{2}$ if the following condition is hold:

$\varphi_{2}(s) \neq 0, \kappa(t) \neq 0, \varphi_{1}(s)>0, \varphi_{1}(s) \vartheta^{2}(t)+\varphi_{2}(s) \kappa^{2}(t)=0 ;$

(k) $E_{3}$ if the following condition is hold:

$\varphi_{2}(s) \neq 0, \kappa(t) \neq 0, \varphi_{1}(s)<0, \varphi_{1}(s) \vartheta^{2}(t)+\varphi_{2}(s) \kappa^{2}(t)=0 ;$ 
(l) $E_{5}$ if one of the following conditions is hold:

1) $\varphi_{2}(s)=0, \varphi_{1}(s)>0$;

2) $\varphi_{1}(s)=0, \kappa(t) \neq 0, \varphi_{2}(s)>0$;

(m) $E_{6}$ if one of the following conditions is hold:

1) $\varphi_{2}(s)=0, \varphi_{1}(s)<0$;

2) $\varphi_{1}(s)=0, \kappa(t) \neq 0, \varphi_{2}(s)<0$;

(n) $E_{7}$ if the following condition is hold: $\varphi_{1}(s)>0, \varphi_{2}(s)>0$

(o) $E_{8}$ if one of the following conditions is hold:

1) $\varphi_{1}(s)<0, \varphi_{2}(s)<0$;

2) $\varphi_{1}(s) \vartheta^{2}(t)+\varphi_{2}(s) \kappa^{2}(t)>0, \varphi_{1}(s) \varphi_{2}(s)<0$;

(p) $E_{9}$ if the following condition is hold: $\varphi_{1}(s) \vartheta^{2}(t)+\varphi_{2}(s) \kappa^{2}(t)<0, \varphi_{1}(s) \varphi_{2}(s)<0 ;$

(q) $E_{10}$ if the following condition is hold: $\varphi_{1}(s)=0, \varphi_{2}(s)=0$;

(r) $E_{11}$ if the following condition is hold: $\varphi_{1}(s)=0, \kappa(t)=0, \varphi_{2}(s)>0$;

(s) $E_{12}$ if the following condition is hold: $\varphi_{1}(s)=0, \kappa(t)=0, \varphi_{2}(s)<0$;

Proof. The proof follows from Lemma 3.

To illustrate the essence of Theorem 3, consider the following example.

Example 3. Let $\eta(t)=\left\{\begin{array}{ll}t+1, & 0 \leq t<6 \\ 0, & t \geq 6\end{array}, \vartheta(t)=\left\{\begin{array}{l}\frac{1}{\sqrt{2}}(t+1), 0 \leq t<2 \\ \sqrt{(t-1)^{2}+4}, 2 \leq t<3 \\ 0, \quad 3 \leq t<6 \\ t-2, \quad t \geq 6\end{array}\right.\right.$ $\kappa(t)=\left\{\begin{array}{ll}0, & 0 \leq t<1 \\ t-3, & 1 \leq t<6 \\ 0, & t \geq 6\end{array}, \varphi_{1}(s)= \begin{cases}-2, & 0 \leq s<3 \\ 0, & 3 \leq s \leq 5 \\ s-5,7, & s>5\end{cases}\right.$ 


$$
\varphi_{2}(s)=\left\{\begin{array}{lc}
-1, & 0 \leq s<1 \\
0, & 1 \leq s<2 \\
1^{\prime}, & 2 \leq s<3 \\
s-4, & s \geq 3
\end{array}\right.
$$

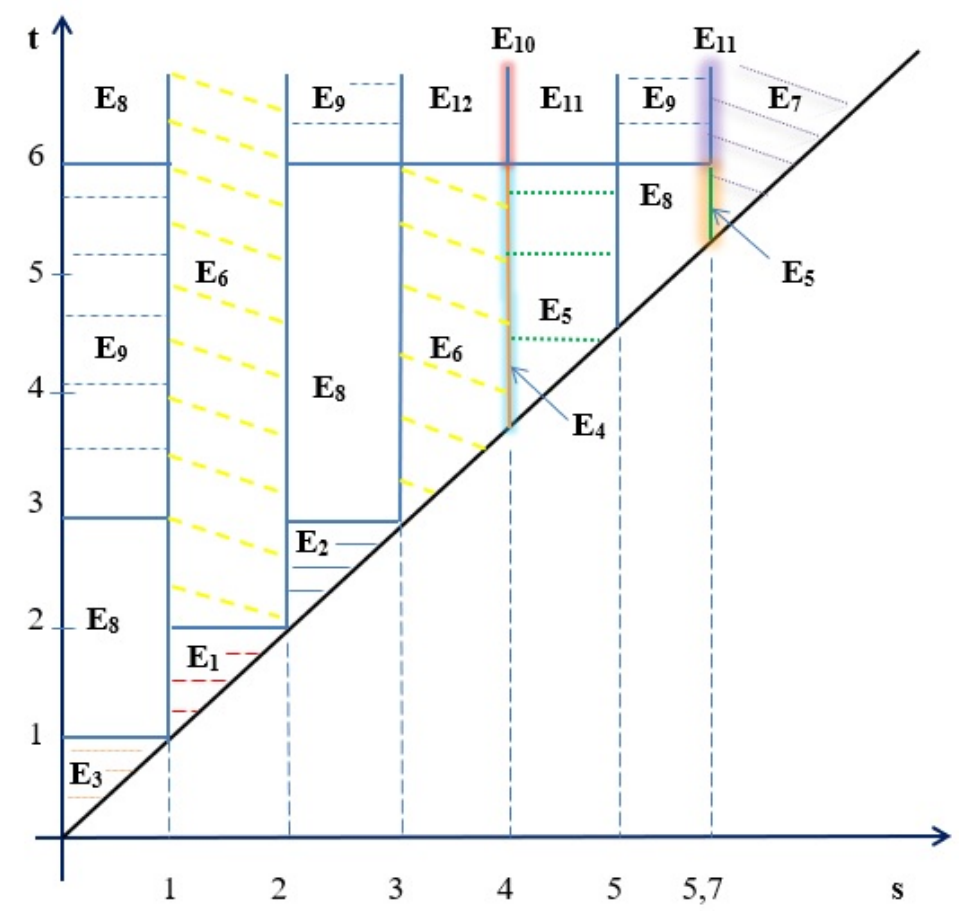

FiguRE 3 . The partition corresponding to the classification of CEA $E_{3}^{[s, t]}$

We consider the next cases (see Fig. 3):

Case 1. If $0 \leq s<1$ then we have

(a) $E_{3}$ when $0 \leq t<1$;

(b) $E_{8}$ when $1 \leq t<3$;

(c) $E_{9}$ when $3 \leq t<6$;

(d) $E_{8}$ when $t \geq 6$;

Case 2. If $1 \leq s<2$ then we have

(a) $E_{1}$ when $1 \leq t<2$;

(b) $E_{6}$ when $t \geq 2$;

Case 3. If $2 \leq s<3$ then we have

(a) $E_{2}$ when $2 \leq t<3$;

(b) $E_{8}$ when $3 \leq t<6$; 
(c) $E_{9}$ when $t \geq 6$;

Case 4. If $3 \leq s<4$ then we have

(a) $E_{6}$ when $3 \leq t<6$;

(b) $E_{12}$ when $t \geq 6$;

Case 5. If $s=4$ then we have

(a) $E_{4}$ when $4 \leq t<6$;

(b) $E_{10}$ when $t \geq 6$;

Case 6. If $4<s \leq 5$ then we have

(a) $E_{5}$ when $4<t<6$;

(b) $E_{11}$ when $t \geq 6$;

Case 7. If $5<s<5,7$ then we have

(a) $E_{8}$ when $5<t<6$;

(b) $E_{9}$ when $t \geq 6$;

Case 8. If $s=5,7$ then we have

(a) $E_{5}$ when $5,7 \leq t<6$;

(b) $E_{11}$ when $t \geq 6$;

Case 9. If $s>5,7$ then we have $E_{7}$ when $t>5,7$.

\section{REFERENCES}

[1] Y.C. Casado, M.S. Molina, M.V. Velasco, Classification of three - dimensional evolution algebras, Linear Algebra Appl., 524 (2017), 68-108.

[2] J.M. Casas, M. Ladra, B.A. Omirov, U.A. Rozikov, On evolution algebras, Algebra Colloq. 21(2) (2014), 331-342.

[3] J.M. Casas, M. Ladra, U.A. Rozikov, A chain of evolution algebras, Linear Algebra Appl., 435(04) (2011), 852-870.

[4] J. M. Casas, M. Ladra, U.A. Rozikov, Markov processes of cubic stochastic matrices: quadratic stochastic processes. Linear Algebra Appl. 575 (2019), 273-298.

[5] A.N. Imomkulov, Behavior and dynamics of the set of absolute nilpotent and idempotent elements of chain of evolution algebras depending on the time, Bulletin National Univ. Uzbekistan: Math. Nat. Sci. 3(4), Article 2 (2020).

[6] A.N. Imomkulov, M.V. Velasco, Chain of three-dimensional evolution algebras, Filomat, 34(10) (2020), 3175-3190.

[7] A.N. Imomkulov, Classification of a family of three dimensional real evolution algebras, TWMS J. Pure Appl. Math., 10(2) (2019), 225-238.

[8] M. Ladra, U.A. Rozikov, Flow of finite-dimensional algebras, Journal of Algebra. 470 (2017), 263-288.

[9] M. Ladra, U.A. Rozikov, Construction of flows of finite-dimensional algebras, Journal of Algebra. 492 (2017), 475-489.

[10] M. Ladra, Sh.N. Murodov, On new classes of chains of evolution algebras. Hacet. J. Math. Stat. 50(1) (2021), 146-158.

[11] B.J. Mamurov, U.A. Rozikov, S.S. Xudayarov, Quadratic stochastic processes of type $(\sigma \mid \mu)$. Markov Processes Related Fields. 26(5) (2020), 915-933.

[12] F. Mukhamedov, O. Khakimov, B. Omirov, I. Qaralleh, Derivations and automorphisms of nilpotent evolution algebras with maximal nilindex. J. Algebra Appl. 18(12) (2019), 1950233, 23 pp.

[13] F. Mukhamedov, O. Khakimov, I. Qaralleh, Classification of nilpotent evolution algebras and extensions of their derivations. Comm. Algebra 48(10) (2020), 4155-4169. 
[14] Sh. Murodov, Classification dynamics of two-dimensional chains of evolution algebras, International Jour. Math. 25(02) (2014), 1450012, (23 pages).

[15] Sh.N. Murodov, Classification of two-dimensional real evolution algebras and dynamics of some twodimensional chains of evolution algebras, Uzb. Mat. Jour. 2 (2014), 102-111.

[16] Sh.N. Murodov, Time depending dynamics of chains of evolution algebras, PhD thesis, Universidad de Santiago de Compostela, (2019).

[17] B. A. Narkuziyev, Some remarks on evolution algebras corresponding to permutations. J. Algebra Appl. https://doi.org/10.1142/S0219498822501122

[18] B.A. Omirov, U.A. Rozikov, K.M. Tulenbayev, On real chains of evolution algebras, Linear and Multilinear Algebra. 63(1) (2015), 586-600.

[19] U.A. Rozikov, Population dynamics: algebraic and probabilistic approach. World Sci. Publ. Singapore. 2020, 460 pp.

[20] U.A. Rozikov, Sh.N. Murodov, Chain of evolution algebras of "chicken" population, Linear Algebra App. 450 (2014), 186-201.

[21] U.A. Rozikov, Sh.N. Murodov, Dynamics of two-dimensional evolution algebras, Lobachevskii Jour. Math. 32(4) (2011), 270-277.

[22] J.P. Tian, Evolution algebras and their applications, Lecture Notes in Mathematics, 1921, SpringerVerlag, Berlin, 2008.

B.A. Narkuziyev, V.I.Romanovskiy Institute of Mathematics of Uzbek Academy of ScIENCES, TASHKENT, UZBEKISTAN.

Email address: bnarkuziev@yandex.ru

U.ROZIKOV ${ }^{a, b, c}$

$a$ V.I.Romanovskiy Institute of Mathematics of UzbeK Academy of Sciences;

${ }^{b}$ AKFa University, 1st Deadlock 10, Kukcha Darvoza, 100095, Tashkent, Uzbekistan;

${ }^{c}$ Faculty of Mathematics, National University of Uzbekistan.

Email address: rozikovu@yandex.ru 